\title{
Frequency Diverse Array Radar Cramér-Rao Lower Bounds for Estimating Direction, Range, and Velocity
}

\author{
Yongbing Wang, ${ }^{1}$ Wen-Qin Wang, ${ }^{1,2}$ and Huaizong Shao ${ }^{1}$ \\ ${ }^{1}$ School of Communication and Information Engineering, University of Electronic Science and Technology of China, \\ Chengdu 611731, China \\ ${ }^{2}$ Department of Electrical and Electronic Engineering, Imperial College London, London SW7 2AZUK, UK
}

Correspondence should be addressed to Yongbing Wang; wangyongbing.hi@163.com

Received 27 January 2014; Accepted 19 February 2014; Published 8 April 2014

Academic Editor: Frankie KitWing Chan

Copyright (C) 2014 Yongbing Wang et al. This is an open access article distributed under the Creative Commons Attribution License, which permits unrestricted use, distribution, and reproduction in any medium, provided the original work is properly cited.

\begin{abstract}
Different from phased-array radar, frequency diverse array (FDA) radar offers range-dependent beampattern and thus provides new application potentials. But there is a fundamental question: what estimation performance can achieve for an FDA radar? In this paper, we derive FDA radar Cramér-Rao lower bounds (CRLBs) for estimating direction, range (time delay), and velocity (Doppler shift). Two different data models including pre- and postmatched filtering are investigated separately. As the FDA radar has range-angle coupling, we use a simple transmit subaperturing strategy which divides the whole array into two subarrays, each uses a distinct frequency increment. Assuming temporally white Gaussian noise and linear frequency modulated transmit signal, extensive simulation examples are performed. When compared to conventional phased-array radar, FDA can yield better CRLBs for estimating the direction, range, and velocity. Moreover, the impacts of the element number and frequency increment are also analyzed. Simulation results show that the CRLBs decrease with the increase of the elements number and frequency increment.
\end{abstract}

\section{Introduction}

The main task of a radar is to detect the existence of targets and estimate their unknown parameters, for example, range, velocity, and direction of arrival (DOA) [1-4]. All the elements in a phased-array antenna transmit the same signal at a fixed carrier frequency. By controlling the linear phase progression across elements, the beam can be steered to the desired direction precisely. But the beam steering is fixed in an angle for all the ranges and cannot mitigate undesirable range-dependent interferences. For this reason, the range and angle information of targets cannot be directly obtained for a conventional phased-array radar, which offers only the angle information. However, in some applications, it is desired that the beam pointing can be steerable to the range of interest.

Recently, frequency diverse array (FDA) is proposed in [5-7] as a method to achieve range-dependent beamforming, which can be used to suppress range-dependent interferences and clutter [8] or improve detection performance
[9]. The most important difference of FDA antenna from a conventional phased-array antenna is that a small amount of frequency increment compared to the carrier frequency is used across the array elements. The periodicity of FDA beampattern in range, angle, and time is studied in [10]. In [11], the FDA is investigated from a simulation perspective and a low-cost FDA is designed. The FDA using chirp (linear frequency modulation) waveforms with different starting frequencies is analyzed in [12] to characterize the associated range-dependent beampattern. The application of FDA in synthetic aperture radar (SAR) is investigated in [13, 14]. Additional studies to exploit the range-dependent beampattern characteristics have been reported in [15].

FDA offers a range-angle-dependent beampattern, which is of great importance as this provides a potential for rangeangle localization of targets. Therefore, this paper considers a fundamental question: what estimation performance can be achieved for an FDA radar? Referring to conventional phased-array range radar, the Cramér-Rao lower bound 
(CRLB) expressions for estimating velocity, direction, and Doppler shift are derived in $[16,17]$. By assuming a narrowband signal model prior to matched filtering, the CRLBs for estimating the time delay, direction, and Doppler shift with a phased-array radar are detailed in $[18,19]$. Furthermore, the CRLB for jointly estimating the attributes of a moving target using MIMO radar is derived in [20-22].

Different from the above literatures, this paper derives the CRLBs for jointly estimating the range, direction, and Doppler shift with an FDA radar. Two typical data models, namely, pre- and postmatched filtering are analyzed separately for static and moving targets, respectively. Since the range and angle of targets cannot be estimated directly due to the range-angle coupling, we use a simple transmit subaperturing strategy for the FDA radar. This method divides the whole array elements into two subarrays; each uses a different frequency increment. In doing so, the range and angle of targets can be solely estimated. The corresponding CRLBs for estimating direction, range, and velocity are derived. Furthermore, the impacts of the elements number and frequency increment on the CRLBs are also investigated.

The rest of the paper is organized as follows. The FDA is briefly introduced in Section 2. In Section 3, we consider the data model after matched filtering and derive the CRLB expressions for estimating the range and direction. In order to decouple the range-angle coupling response, we divide the whole array into two subarrays. The corresponding CRLBs are derived. In Section 4, the data model prior to matched filtering is investigated and the CRLB expressions for estimating the direction, range, and Doppler shift are derived, where temporally white Gaussian noise and chirp pulse signal are assumed. Finally, extensive simulation results are provided in Section 5. This paper is concluded in Section 6.

\section{Frequency Diverse Array (FDA)}

In conventional phased-array radars, it is assumed that an identical waveform is radiated from each element, excluding the amplitudes and phases. Different from phased-array radars, FDA elements can be either excited by the same waveform or different waveforms. For simplicity and without loss of generality, we consider a uniform linear array (ULA) FDA and assume that the waveforms radiated from each antenna element are identical but with a frequency increment of $\Delta f \mathrm{~Hz}$. That is, the radiation frequency of the $m$ th element is

$$
f_{m}=f_{0}+(m-1) \cdot \Delta f, \quad m=0,1, \ldots, M-1,
$$

where $f_{0}$ is the radar carrier frequency and $M$ is the number of the elements.

Supposing a narrowband transmit signal and taking the first element as the reference for the array, the steering vector is given by the following equation [11]:

$$
\mathbf{a}(\theta, r)=\left[\begin{array}{lllll}
1 & e^{-j\left(\left(2 \pi f_{0} d \sin \theta / c\right)+((2 \pi \Delta f \cdot d \sin \theta) / c)-(2 \pi r \Delta f / c)\right)} & \cdots & e^{-j\left(\left(\left(2 \pi f_{0}(M-1) d \sin \theta\right) / c\right)+\left(\left(2 \pi \cdot(M-1)^{2} \Delta f \cdot d \sin \theta\right) / c\right)-((2 \pi r(M-1) \Delta f) / c)\right)}
\end{array}\right]^{T},
$$

where $\theta$ is the direction, $r$ is the range, $d$ is the element spacing, $c$ is the speed of light, and $T$ is the transpose. Since $f_{0} \gg \Delta f$ and $r \gg(M-1) d \sin (\theta)$, in an amplitude sense an approximation can be taken by ignoring the phase term $2 \pi \cdot(m-1)^{2} \Delta f \cdot d \sin (\theta) / c$ because it has ignorable impacts. In this case, the steering vector can be simplified to

$$
\mathbf{a}(\theta, r) \approx\left[\begin{array}{llll}
1 & e^{-j\left(\left(2 \pi f_{0} d \sin (\theta) / c\right)-((2 \pi \cdot \Delta f \cdot r) / c)\right)} & \cdots & e^{-j\left(\left(\left(2 \pi f_{0}(m-1) d \sin (\theta)\right) / c\right)-((2 \pi \cdot(m-1) \Delta f \cdot r) / c)\right)}
\end{array}\right]^{T} .
$$

The FDA characteristics can be summarized as follows [7-12]. (1) If the frequency offset $\Delta f$ is fixed, the beam direction will vary as a function of the range $r$; that is, it is a range- dependent beam. (2) If the range is $r$ fixed, the beam direction will vary as a function of $\Delta f$. This means that the FDA is frequency-increment-dependent (3) If the frequency increment across the array is not applied (i.e., $\Delta f=0$ ), the corresponding FDA is just a conventional ULA phased array.

\section{CRLB with Signal Model after Matched Filtering}

3.1. Data Models. Suppose that a target is located at $(\theta, r)$. After matched filtering, the baseband equivalent of the complex valued signals at the receiver can be expressed as

$$
\mathbf{y}=\beta_{0} \mathbf{a}(\theta, r)+\mathbf{n}=\vec{\mu}(\psi)+\mathbf{n},
$$


where $\beta_{0}$ is a constant for a given $\operatorname{target}, \vec{\mu}(\psi)=\beta_{0} \mathbf{a}(\theta, r)$ with $\psi=[\theta, r]^{T}$, and $\mathbf{n}$ is a zero-mean complex Gaussian white noise with spatial covariance:

$$
E\left[\mathbf{n n}^{\mathrm{H}}\right]=\mathbf{R}_{\mathbf{n}}=\sigma_{n}^{2} \mathbf{I}_{M},
$$

where $E[\cdot]$ is the expectation operator, $\mathrm{H}$ is the conjugate transpose, $\mathbf{R}_{\mathbf{n}}$ is the spatial noise covariance matrix, $\sigma_{n}^{2}$ is the noise power, and $\mathbf{I}_{M}$ is an $M \times M$ identity matrix.

3.2. CRLBs of Angle and Range Estimations. In the following, we discuss the CRLBs for several different cases separately.

3.2.1. Range Is Known and Angle Is Unknown. In this case, $\psi=\theta$. The corresponding Fisher information matrix (FIM) is $\mathbf{I}_{\psi \psi}[23]$ :

$$
\mathbf{I}_{\psi \psi}=2 \operatorname{Re}\left\{D_{\psi_{i}}^{\mathrm{H}}(\psi)\left(\mathbf{R}_{\mathbf{n}}^{-1}\right) D_{\psi_{j}}(\psi)\right\},
$$

where $\operatorname{Re}\{\cdot\}$ is the real part of the signal, $\psi_{i}$ is the $i$ th element of $\psi$, and the $D_{\psi_{i}}(\psi)$ is [24]

$$
D_{\psi_{i}}(\psi)=\frac{\partial \vec{\mu}(\psi)}{\partial \psi_{i}} .
$$

Under the signal model (4), the FDA FIM with respect to $\theta$ is expressed as

$$
\begin{aligned}
& I_{\theta \theta_{\mathrm{FDA}}}= 8 \pi^{2} d^{2} \cos ^{2}(\theta) \\
& \cdot \operatorname{SNR}\left\{\frac{\sum_{m=1}^{M}(m-1)^{2}}{\lambda^{2}}+\frac{(\Delta f)^{2} \sum_{m=1}^{M}(m-1)^{4}}{c^{2}}\right. \\
&\left.+\frac{2 \Delta f \sum_{m=1}^{M}(m-1)^{3}}{\lambda c}\right\},
\end{aligned}
$$

where SNR is the signal-to-noise ratio (SNR). Accordingly, the CRLB is

$$
\mathrm{CRLB}_{\theta \theta_{\mathrm{FDA}}}=I_{\theta \theta_{\mathrm{FDA}}}^{-1},
$$

where -1 denotes the inverse matrix. In particular, when $\Delta f=0$, it is simplified to

$$
I_{\theta \theta_{\text {phased-array }}}=8 \operatorname{SNR} \frac{\pi^{2} d^{2} \cos ^{2}(\theta)}{\lambda^{2}} \sum_{m=1}^{M}(m-1)^{2} .
$$

Proof. See Appendix A.

It can be easily proved that

$$
\mathrm{CRLB}_{\theta \theta_{\mathrm{FDA}}}<\mathrm{CRLB}_{\theta \theta_{\text {phased-array }}} .
$$

That is to say, the FDA radar has better CRLB for angle estimation than phased-array radar.
3.2.2. Angle Is Known and Range Is Unknown. As phasedarray radar has range-independent beam, here we only calculate the range estimation for FDA radar. The FIM with respect to $r$ can be expressed as

$$
I_{r r_{\mathrm{FDA}}}=2 \mathrm{SNR} \frac{4 \pi^{2}(\Delta f)^{2}}{c^{2}} \sum_{m=1}^{M}(m-1)^{2} .
$$

The corresponding CRLB is

$$
\mathrm{CRLB}_{r r_{\mathrm{FDA}}}=I_{r r_{\mathrm{FDA}}}^{-1} .
$$

Proof. See Appendix B.

3.2.3. Both Angle and Range Are Unknown. In this case, the $\theta$ and $r$ CRLBs can be similarly determined as

$$
\begin{aligned}
\mathrm{CRLB}_{\theta \theta_{\mathrm{FDA}}}= & \left(c^{2} \cdot\left(\sum_{m=1}^{M}(m-1)^{2}\right)\right) \\
& \times\left(2 \mathrm{SNR} \cdot 4 \pi^{2} d^{2} \cdot(\Delta f)^{2} \cos ^{2}(\theta)\right. \\
& \times\left(\sum_{m=1}^{M}(m-1)^{4} \sum_{m=1}^{M}(m-1)^{2}\right. \\
\mathrm{CRLB}_{r r_{\mathrm{FDA}}}= & \left.\left(c^{4} \cdot\left(\frac{\sum_{m=1}^{M}(m-1)^{2}}{\lambda^{2}} \sum_{m=1}^{M}(m-1)^{3}\right)^{2}\right)\right)^{-1} \\
& +\frac{(\Delta f)^{2} \sum_{m=1}^{M}(m-1)^{4}}{c^{2}} \\
& \left.\times\left(\frac{2 \Delta f \sum_{m=1}^{M}(m-1)^{3}}{\lambda c}\right)\right)^{2 S N R} \cdot 4 \pi^{2} \cdot(\Delta f)^{4} \\
& \times\left(\sum_{m=1}^{M}(m-1)^{4} \sum_{m=1}^{M}(m-1)^{2}\right. \\
& \left.\left.-\left(\sum_{m=1}^{M}(m-1)^{3}\right)^{2}\right)\right)^{-1}
\end{aligned}
$$

Proof. See Appendix C.

When the target's range and angle are estimated jointly, the range and angle CRLBs will be significantly degraded due to the range-angle coupling (which will be further investigated in Section 5). Consequently the range and angle 


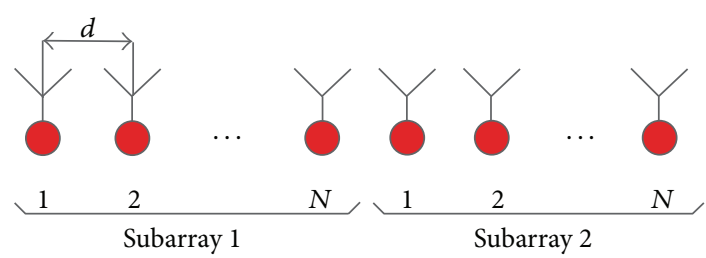

FIGURE 1: Illustration of transmit subaperturing FDA radar.

of targets cannot be estimated directly by a standard FDA radar. To overcome this problem, we present a transmit subaperturing method for the FDA radar.
3.2.4. CRLBs of Transmit Subaperturing FDA Radar. To decouple the range and angle peaks and estimate both the range and angle of target, we divide the whole array into two equal subarrays $[25,26]$. Suppose that the number of elements is $M$; each subarray has $N$ elements; namely, $M=2 N$. The first subarray uses the frequency increment of $\Delta f_{1}$, and the second subarray uses the frequency increment of $\Delta f_{2}$, as shown in Figure 1. The resulting system is referred to as transmit subaperturing FDA (TS-FDA) radar.

Taking the first element of the first subarray as the reference, the new steering vector can be represented as the following equation:

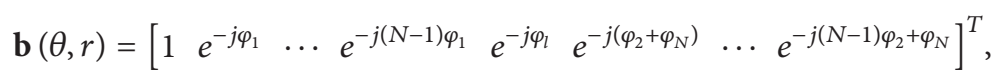

where

$$
\begin{aligned}
\varphi_{1} & =\left(\frac{2 \pi d \sin (\theta)}{\lambda}\right)-\left(\frac{2 \pi r \cdot \Delta f_{1}}{c}\right), \\
\varphi_{N} & =\left(\frac{2 \pi N d \sin (\theta)}{\lambda}\right), \\
\varphi_{2} & =\left(\frac{2 \pi d \sin (\theta)}{\lambda}\right)-\left(\frac{2 \pi r \cdot \Delta f_{2}}{c}\right) .
\end{aligned}
$$

The corresponding FIM can be derived as

$$
\mathbf{I}_{\mathrm{TS}-\mathrm{FDA}}=2 \operatorname{Re}\left\{D_{\psi_{i}}^{\mathrm{H}}(\psi)\left(\mathbf{R}_{\mathbf{n}}^{-1}\right) D_{\psi_{j}}(\psi)\right\}=\left[\begin{array}{cc}
I_{\theta \theta} & I_{\theta r} \\
I_{r \theta} & I_{r r}
\end{array}\right],
$$

where

$$
\begin{gathered}
I_{\theta \theta}=2 \frac{\left|\beta_{0}\right|^{2}}{\sigma_{n}^{2}} \frac{4 \pi^{2} d^{2} \cos ^{2}(\theta)}{\lambda^{2}} \sum_{m=1}^{2 N}(m-1)^{2} \\
I_{r r}=2 \frac{\left|\beta_{0}\right|^{2}}{\sigma_{n}^{2}} \frac{4 \pi^{2}\left(\Delta f_{1}^{2}+\Delta f_{2}^{2}\right)}{c^{2}} \sum_{m=1}^{N}(m-1)^{2} \\
I_{\theta r}=I_{r \theta}=-\frac{2\left|\beta_{0}\right|^{2}}{\sigma_{n}^{2}} \frac{4 \pi^{2} d \cos (\theta)}{\lambda c} \\
\times\left[\left(\Delta f_{1}+\Delta f_{2}\right) \sum_{m=1}^{N}(m-1)^{2}+\Delta f_{2} N \sum_{m=1}^{N}(m-1)\right]
\end{gathered}
$$

The angle and range CRLBs are the first two diagonal elements of the inverse of the FIM

$$
\begin{aligned}
& \operatorname{CRLB}_{\theta \theta_{\text {TS-FDA }}}=\left[\mathbf{I}_{\mathrm{TS}-\mathrm{FDA}}^{-1}\right]_{1,1}, \\
& \mathrm{CRLB}_{r r_{\mathrm{TS}-\mathrm{FDA}}}=\left[\mathbf{I}_{\mathrm{TS}-\mathrm{FDA}}^{-1}\right]_{2,2},
\end{aligned}
$$

where $[\cdot]_{i, j}$ is the element at the $i$ th row and $j$ th column of the matrix.

\section{CRLB with Signal Model prior to Matched Filtering}

4.1. Data Models. Unlike the first data model, the data model prior to matched filtering allows to estimate the Doppler shift (velocity). Suppose an $M$-element antenna array receives a time delayed and Doppler-shifted echo of the transmitted signal $s(t) \exp \left(j \Omega_{c} t\right)$, where $\Omega_{c}$ is the center frequency. Knowing the time delay and Doppler shift $\Omega_{D}$ (assuming a target with constant radial velocity), the range and radial component of velocity can be determined by $r=c \tau / 2$ and $v=\Omega_{D} c /\left(2 \Omega_{c}\right)$. We denote the continuous-time signal $s(t)$ as $s[l]=s(l \cdot \Delta t)$, where $\Delta t$ is the sampling interval. Correspondingly, the time delay and Doppler shift in the sampled signal domain are $l_{\tau}=\tau / \Delta t$ and $w_{D}=\Omega_{D} \cdot \Delta t$, respectively [27].

After converting to baseband and sampling, the received signal at time $l \cdot \Delta t$ becomes

$\mathbf{y}[n]=\beta \cdot \mathbf{a}(\theta, r) \cdot s\left[l-l_{\tau}\right] \exp \left(j w_{D} l\right)+\mathbf{n}[l], \quad l=1, \ldots, L$,

where $\beta$ is complex amplitude of the signal and $\mathbf{n}[l]$ is the additive noise [28].

We assume that the snapshots taken at $l=1, \ldots, L$ cover the whole of a coherent processing interval (CPI). Therefore, the time duration of the CPI is $T_{\mathrm{CPI}}=L \cdot \Delta t$. Under the data model of (21), the complex amplitude $\beta$ is assumed to be an unknown deterministic constant during the CPI. To model the Doppler effect with a frequency shift, the radial component of the target velocity needs to be much smaller than the propagation speed (i.e., $v / c \ll 1$ ). Then the timebandwidth product of the complex envelope should be larger than 1 . In addition, it is assumed that the propagation time of the signal across the array is much smaller than the reciprocal 
of the signal bandwidth, which is the narrowband array assumption in array processing.

Define the vector of unknown target parameters as $\kappa=\left[\operatorname{Re}\{\beta\}, \operatorname{Imag}\{\beta\}, \theta, \eta^{T}\right]$. Stacking all samples into a single vector, (21) can be rewritten as

$$
\mathbf{z}=\beta \cdot \vec{\phi}(\eta) \otimes \mathbf{a}(\theta, r)+\mathbf{n}=\vec{\mu}(\kappa)+\mathbf{n},
$$

where $\otimes$ denotes the Kronecker product, $\vec{\mu}(\kappa)=$ $\beta \cdot \vec{\phi}(\eta) \otimes \mathbf{a}(\theta, r)$.

$$
\begin{aligned}
\vec{\phi}(\eta)= & {\left[s\left[1-\frac{2 r}{c \cdot \Delta t}\right] \exp \left(j w_{D} 1\right),\right.} \\
& s\left[2-\frac{2 r}{c \cdot \Delta t}\right] \exp \left(j w_{D} 2\right), \ldots, \\
& \left.s\left[L-\frac{2 r}{c \cdot \Delta t}\right] \exp \left(j w_{D} L\right)\right]^{T} .
\end{aligned}
$$

The noise $\mathbf{n}$ is assumed to be zero-mean Gaussian, spatially and temporally correlated with spatiotemporal covariance [29]

$$
E\left[\mathbf{n n}^{\mathrm{H}}\right]=\mathbf{C}_{\mathbf{n}} \otimes \mathbf{R}_{\mathbf{n}}
$$

where $\mathbf{C}_{\mathbf{n}}$ is the temporal noise covariance matrix.

Under the above data model, the signal and noise parameters are disjoint and satisfy the space-time separability [30].

4.2. CRLBs of Angle, Range, and Doppler Shift. Suppose the received signal is completely covered by the observations $l=1, \ldots, L$ and the sampling is dense (i.e., $\Delta t \rightarrow 0$ ). The $\mathbf{R}_{\mathbf{n}}$ is assumed to be constant in the frequency band $f \in$ $(-1 /(2 \Delta t), 1 /(2 \Delta t))$, where $f$ denotes the frequency in the continuous-time domain. The vector of Doppler shift and time delay in the continuous-time domain is defined as

$$
\eta=\left[r, \Omega_{D}\right]^{T}
$$

For $\mathbf{C}_{\mathbf{n}}=\mathbf{I}_{L}$ and $\mathbf{R}_{\mathbf{n}}=\sigma_{n}^{2} \mathbf{I}_{M}$ with $\mathbf{I}_{L}$ being an $L \times L$ identity matrix. We define the signal power $\|\mathbf{s}\|=\vec{\phi}^{\mathrm{H}} \vec{\phi}$. The CRLBs expression for $\theta$ and $\eta$ follows from Appendix D as the following:

$$
\begin{aligned}
& \operatorname{CRLB}_{\theta \eta, \theta \eta_{\mathrm{FDA}}} \\
& =\frac{\sigma_{n}^{2}}{2|\beta|^{2}\|\boldsymbol{s}\|} \\
& \quad \times\left[\begin{array}{ccc}
\pi^{2} d^{2} \cos ^{2}(\theta) M\left(M^{2}-1\right)\left[\frac{1}{3 \lambda^{2}}+\frac{(\Delta f)^{2}\left[(2 M-1)\left(8 M^{2}-3 M-11\right)\right]}{45 c^{2}(M+1)}+\frac{2 \Delta f(M-1)}{3 c \lambda}\right]-\pi^{2} d \Delta f \cos (\theta) M\left(M^{2}-1\right)\left[\frac{1}{3 \lambda c}+\frac{\Delta f(M-1)}{3 c^{2}}\right] & \pi^{2}(\Delta f)^{2} \frac{M\left(M^{2}-1\right)}{3 c^{2}}+\frac{\xi_{1,1}}{\|\boldsymbol{s}\|} \\
-\pi^{2} d \Delta f \cos (\theta) M\left(M^{2}-1\right)\left[\frac{1}{3 \lambda c}+\frac{\Delta f(M-1)}{3 c^{2}}\right] & \frac{\operatorname{Imag}\left\{\xi_{1,2}\right\}}{\|\boldsymbol{s}\|} & \frac{\xi_{2,2}}{\|\boldsymbol{s}\|}
\end{array}\right],
\end{aligned}
$$

where

$$
\begin{aligned}
& \xi_{1,1}= \int_{-\infty}^{\infty}\left|\frac{d s(t-\tau)}{d t}\right|^{2} d t \\
&-\frac{1}{\|\mathbf{s}\|}\left[\int_{-\infty}^{\infty} s(t-\tau) \frac{d s(t-\tau)^{\mathrm{H}}}{d t} d t\right]^{2}, \\
& \xi_{2,2}= \int_{-\infty}^{\infty} t^{2}|s(t-\tau)|^{2} d t \\
&-\frac{1}{\|\mathbf{s}\|}\left[\int_{-\infty}^{\infty} t|s(t-\tau)|^{2} d t\right]^{2}, \\
& \xi_{1,2}= \int_{-\infty}^{\infty} t s(t-\tau) \frac{d s(t-\tau)^{\mathrm{H}}}{d t} d t \\
&-\left[\frac{1}{\|\mathbf{s}\|} \int_{-\infty}^{\infty} t|s(t-\tau)|^{2} d t\right. \\
&\left.\cdot \int_{-\infty}^{\infty} s(t-\tau) \frac{d s(t-\tau)^{\mathrm{H}}}{d t} d t\right] .
\end{aligned}
$$

The terms $\xi_{1,1}$ and $\xi_{2,2}$ are proportional to the root mean squared (RMS) bandwidth and RMS duration of $s(t)$, respectively. Note that the decoupling is a consequence of the assumed space-time separability of signal and noise models and the assumption of the complex amplitude $\beta$ as an unknown deterministic constant. In addition, since the signal and additive noise parameters are disjoint, the above CRLB expressions hold regardless of whether the spatiotemporal noise covariance $\mathbf{C}_{\mathbf{n}} \otimes \mathbf{R}_{\mathbf{n}}$ is known or unknown.

4.3. CRLBs When Chirp Pulse Signal Is Employed. In this section, we derive the CRLB expressions for the range, Doppler shift, and direction estimation when $s(t)$ is a chirp pulse signal with a large time-bandwidth product $[31,32]$. It can be expressed as

$$
s(t)=\sum_{p=0}^{P-1} s_{0}\left(t-p T_{p}\right),
$$


where $T_{p}$ is the pulse repetition interval, $P$ is the number of chirp pulses, and $s_{0}(t)$ is expressed as [12]

$$
s_{0}(t)=\exp \left[j \pi \frac{B}{T_{0}}\left(t-\frac{1}{2} T_{0}\right)^{2}\right] \cdot\left[h(t)-h\left(t-T_{0}\right)\right],
$$

where $T_{0}$ is the chirp pulse duration, $B$ is the chirp bandwidth, and $h(t)$ is the Heaviside step function.
Assume the time-bandwidth product of the pulse is $T_{0} \cdot B \gg 1$. Using the signal given in (29) and (30), in continuous-time domain, we obtain the signal power $\|\mathbf{s}\|=$ $P T_{0}, \xi_{1,1}=P 4 \pi^{2} B^{2} T_{0} / 3 c^{2}, \operatorname{Imag}\left\{\xi_{1,2}\right\}=-\left(P \pi B T_{0}^{2} / 3 c\right)$, and $\xi_{2,2}=\left(P \mathrm{MT}_{0}^{3} / 12\right)\left[1+\left(T_{0} / T_{R}\right)^{2}\left(P^{2}-1\right)\right]$. Thus, the CRLB expressions of $\theta$ and $\eta$ for FDA are derived as the following equation:

$$
\begin{aligned}
& \operatorname{CRLB}_{\theta \eta, \theta \eta_{\mathrm{FDA}}} \\
& =\frac{1}{2 \mathrm{SNR}} \\
& {\left[\begin{array}{ccc}
\pi^{2} d^{2} \cos ^{2}(\theta) M\left(M^{2}-1\right)\left[\frac{1}{3 \lambda^{2}}+\frac{(\Delta f)^{2}\left[(2 M-1)\left(8 M^{2}-3 M-11\right)\right]}{45 c^{2} M(M+1)}+\frac{2 \Delta f(M-1)}{3 c \lambda}\right] & -\pi^{2} d \Delta f \cos (\theta) M\left(M^{2}-1\right)\left[\frac{1}{3 \lambda c}+\frac{\Delta f(M-1)}{3 c^{2}}\right] & 0 \\
-\pi^{2} d \Delta f \cos (\theta) M\left(M^{2}-1\right)\left[\frac{1}{3 \lambda c}+\frac{\Delta f(M-1)}{3 c^{2}}\right] & \pi^{2}(\Delta f)^{2} \frac{M\left(M^{2}-1\right)}{3 c^{2}}+\frac{4 \pi^{2} B^{2} M}{3 c^{2}} & -\frac{M \pi B T_{0}}{3 c} \\
0 & -\frac{M \pi B T_{0}}{3 c} & \frac{M T_{0}^{2}}{12}\left[1+\left(\frac{T_{R}}{T_{0}}\right)^{2}\left(P^{2}-1\right)\right]
\end{array}\right]^{-1}}
\end{aligned}
$$

Specially, for the phased array (i.e., $\Delta f=0$ ), the CRLB is

$$
\begin{aligned}
& \mathrm{CRLB}_{\theta \eta, \theta \eta_{\text {phased-array }}}=\frac{1}{2 \mathrm{SNR}} \\
& \times\left[\begin{array}{ccc}
\frac{\pi^{2} d^{2} \cos ^{2}(\theta) M\left(M^{2}-1\right)}{3 \lambda^{2}} & 0 & 0 \\
0 & \frac{4 \pi^{2} B^{2} M}{3 c^{2}} & -\frac{M \pi B T_{0}}{3 c} \\
0 & -\frac{M \pi B T_{0}}{3 c} & \frac{M T_{0}^{2}}{12}\left[1+\left(\frac{T_{R}}{T_{0}}\right)^{2}\left(P^{2}-1\right)\right]
\end{array}\right]^{-1} .
\end{aligned}
$$

When only one pulse $(P=1)$ is used, the CRLB in (32) will be infinite because the model is not identifiable and the range and Doppler shift cannot be uniquely estimated [18], since phase-array radar has no range identity capability. In contrast, (31) does not have such a problem.
According to the previous discussion, the angle and range of targets cannot be estimated jointly due to the range-angle coupling. Using the similar transmit subaperturing approach presented in Section 3 and steering vector $\mathbf{b}(\theta, r)$ (15), the CRLBs of angle, range, and Doppler shift are derived as in the following equation:

$$
\mathrm{CRLB}_{\theta \eta, \theta \eta_{\mathrm{TS}-\mathrm{FDA}}}=\frac{1}{2 \mathrm{SNR}}
$$

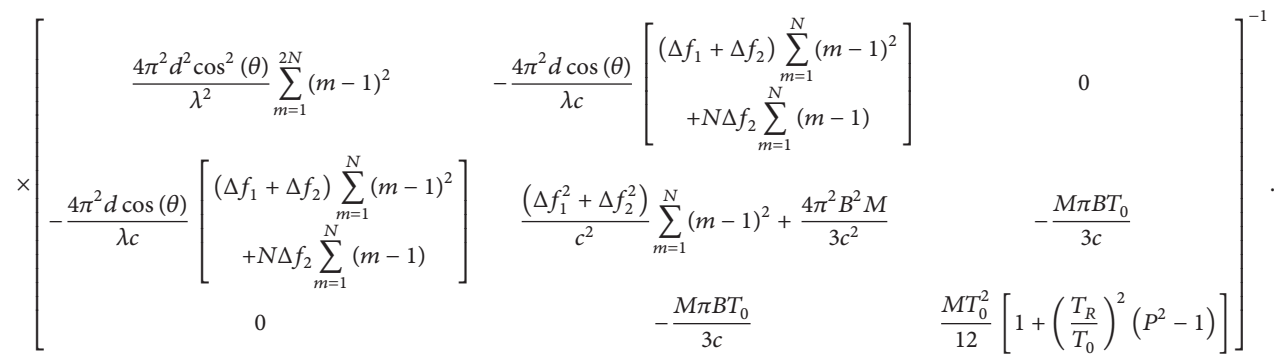




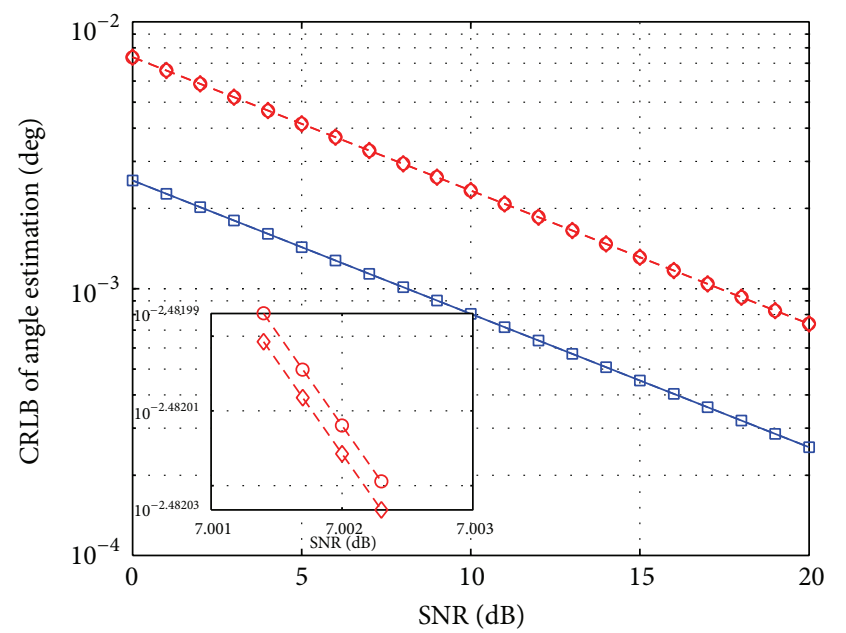

$$
\begin{aligned}
& -\ominus-M=16, \Delta f=15 \mathrm{kHz} \quad-M=32, \Delta f=15 \mathrm{kHz} \\
& -\vartheta-M=16, \Delta f=30 \mathrm{kHz} \quad \text { - в- } M=32, \Delta f=30 \mathrm{kHz}
\end{aligned}
$$

FIGURE 2: CRLB for estimating angle versus SNR when the range is known.

\section{Simulation and Verification}

In this section, we consider several numerical examples that compare the CRLBs in different signal and noise models. Consider an X-band FDA radar with the carrier frequency $f_{0}=10 \mathrm{GHz}$. We assume a ULA of $M$ elements used for transmitting. The array elements are spaced half of the wavelength apart from each other; namely, $d=\lambda / 2$. One target of interest is supposed to reflect a plane wave that impinges on the array from direction of angle $\theta=30^{\circ}$.

Under the signal model after matched filtering, Figures 2 and 3 compare the CRLBs according to (9) and (13), respectively. It can be noticed that the CRLBs are improved when a larger number of elements are employed. However, it has no significant difference when different frequency increments are used. This is because $\sum_{m=1}^{M}(m-1)^{2} / \lambda^{2} \gg$ $(\Delta f)^{2} \sum_{m=1}^{M}(m-1)^{4} / c^{2}+2 \Delta f \sum_{m=1}^{M}(m-1)^{3} / \lambda c$; thus the frequency increment has a small impact on the CRLBs. In [33], a frequency offset selection strategy is derived to precisely steer the beam toward a fixed range with a desired angle.

Figure 4 shows the CRLBs of angle and range when both the angle and range are unknown. The CRLBs are significantly degraded due to the range-angle coupling. Consequently the range and angle of targets cannot be estimated directly by the FDA radar. However, the CRLBs decrease as the increase of the number of elements and frequency increment still holds. Moreover, generally more elements mean that better CRLBs performance can be achieved for the FDA radar.

To overcome the problem that the range and angle of targets cannot be estimated directly by the FDA radar, we use the transmit subaperturing strategy on the transmit frequency increments. Figure 5 shows the corresponding

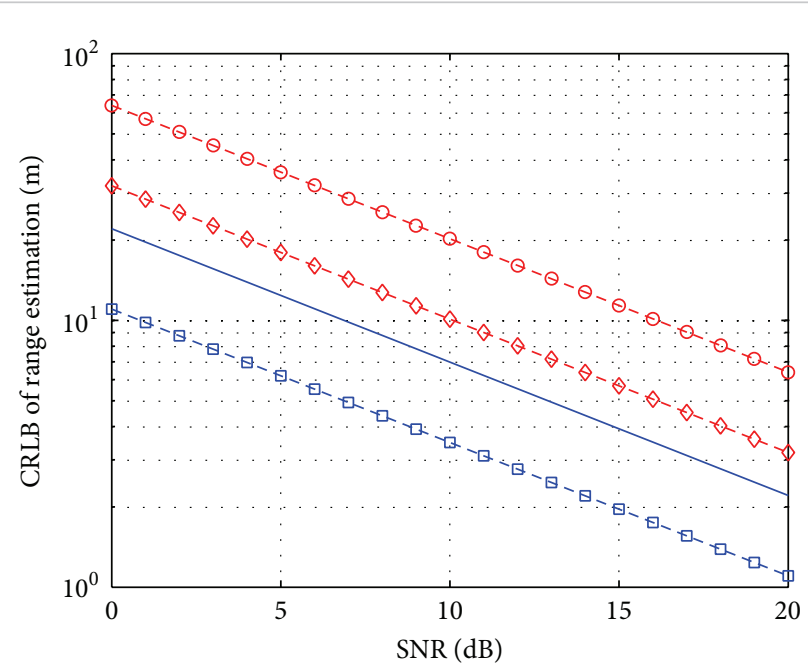

$$
\begin{array}{rlrl}
-\ominus-M & =16, \Delta f=15 \mathrm{kHz} & -M & =32, \Delta f=15 \mathrm{kHz} \\
-\vartheta-M & =16, \Delta f=30 \mathrm{kHz} & - \text { - }-M=32, \Delta f=30 \mathrm{kHz}
\end{array}
$$

FIGURE 3: CRLB for estimating range versus SNR when the angle is known.

CRLBs where $M=32$ is assumed. It can be noticed that, to obtain a lower CRLB, the $\Delta f_{1}$ and $\Delta f_{2}$ should have inverse signs; that is, one is passive and the other is negative. One reason is that, in this case, the FDA radar has a wider system bandwidth. Figure 6 shows the CRLBs of angle and range when $M=20$ is employed. It can be noticed that the CRLBs performance improves with the increase of the sensor number.

Under the data model prior to matched filtering, we suppose the following signal parameters: bandwidth $B=$ $10 \mathrm{MHz}$, repetition period $T_{p}=1 \mathrm{~ms}$, and pulse duration $T_{0}=250 \mu \mathrm{s}$. In this case, the approximate expressions given in (31) are valid because the transmitted has a large time-bandwidth product $\left(T_{0} \cdot B=2500 \gg 1\right)$. Figure 7 shows the CRLBs for direction, range, and Doppler shift as a function of SNR. Note that, when SNR $=-10 \mathrm{~dB}$ and $M=32$ are employed, we can get $\mathrm{CRLB}_{r r_{\mathrm{FDA}}}=6.53 \mathrm{~m}$ and $\mathrm{CRLB}_{\Omega_{D} \Omega_{\mathrm{DFDA}}}=13.6937 \mathrm{rad} / \mathrm{s}$ that corresponds to the CRLB for velocity is $3.27 \mathrm{~cm} / \mathrm{s}$ (since $v=\Omega_{D} c /\left(2 \Omega_{c}\right)$ and $\left.\Omega_{c}=2 \pi f_{c}\right)$. Since $(\Delta f)^{2} \sum_{m=1}^{M}(m-1)^{4} / c^{2} \ll 1$, the frequency increment has a small impact on the CRLBs. In addition, observe that the CRLB for $\theta$ and $\eta$ is block-diagonal (see (32)) and therefore decoupled; that is, $\mathrm{CRLB}_{\eta \eta_{\mathrm{FDA}}}$ remains the same whether or not $\theta$ is known, and similarly, $\mathrm{CRLB}_{\theta \theta_{\mathrm{FDA}}}$ is the same whether or not $\eta$ is known. The decoupling is a consequence of the assumed space-time separability of signal and noise models and the assumption of the complex amplitude $\beta$ as an unknown deterministic constant.

Figure 8 shows that the CRLBs versus SNR for different combinations of $\Delta f_{1}$ and $\Delta f_{2}$. Comparing Figures 8 and 7, the CRLBs have been significantly improved. Likewise, comparing Figures 8 and 9, the CRLBs performance improves as the number of elements increases. 


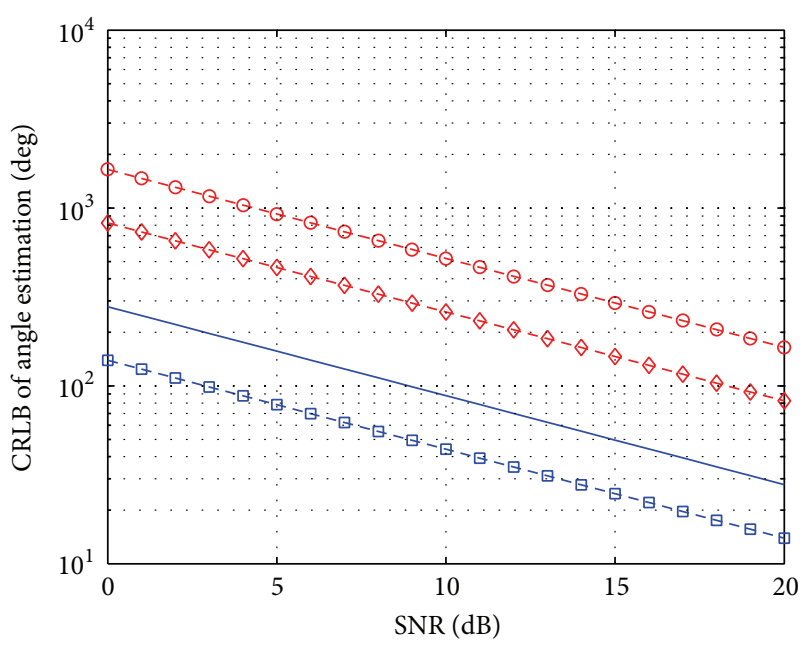

$\begin{array}{ll}-\ominus-M=16, \Delta f=15 \mathrm{kHz} & -M=32, \Delta f=15 \mathrm{kHz} \\ -\diamond-M=16, \Delta f=30 \mathrm{kHz} & - \text { - } M=32, \Delta f=30 \mathrm{kHz}\end{array}$

(a) CRLB for estimating angle versus SNR

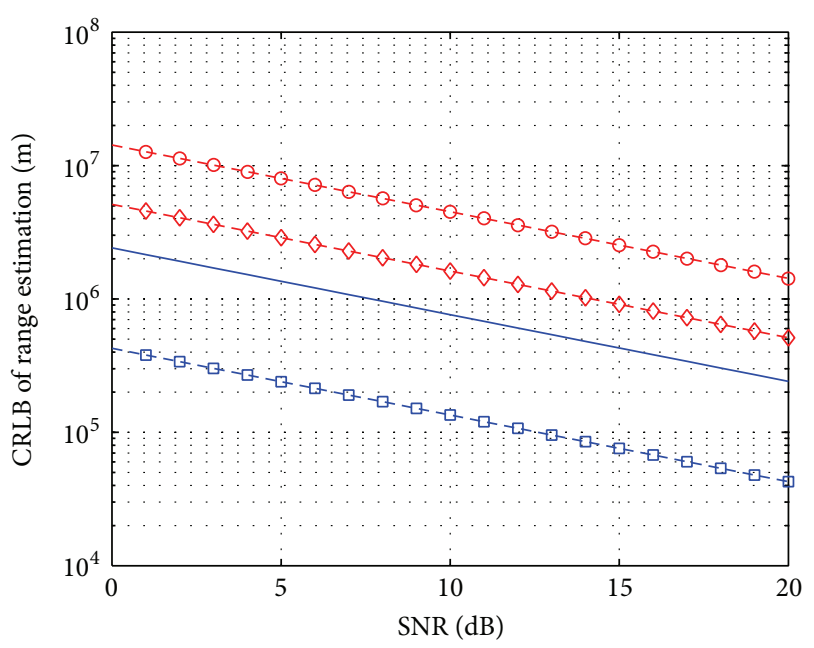

$-\ominus-M=16, \Delta f=15 \mathrm{kHz} \quad-M=32, \Delta f=15 \mathrm{kHz}$

(b) CRLB for estimating range versus SNR

Figure 4: Both angle and range are unknown.

\section{Conclusion}

In this paper, we derive the CRLB to jointly estimate the attributes of a moving target using FDA radar and compute the corresponding CRLB expressions. First, we briefly introduce the FDA concept and make a summary on the FDA characteristics. Then, we consider two different data models, namely, pre- and postmatched filtering. Under different signal and noise models, we compute the CRLB expressions for estimating the range, direction, and Doppler shift. We demonstrate that the FDA radar beamforming is coupled in range and angle and that the target's range and angle cannot be estimated directly by the FDA radar. To overcome

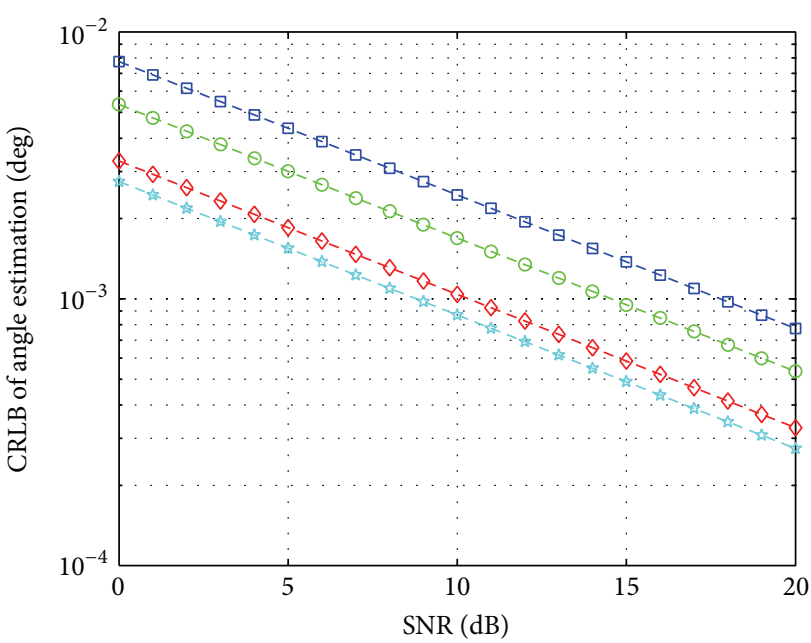

一它- $\Delta f_{1}=-30 \mathrm{kHz}, \Delta f_{2}=30 \mathrm{kHz} \quad-\ominus-\Delta f_{1}=0 \mathrm{kHz}, \Delta f_{2}=30 \mathrm{kHz}$ $-\diamond-\Delta f_{1}=-15 \mathrm{kHz}, \Delta f_{2}=30 \mathrm{kHz} \quad-\boxminus-\Delta f_{1}=15 \mathrm{kHz}, \Delta f_{2}=40 \mathrm{kHz}$

(a) CRLB for estimating angle versus SNR

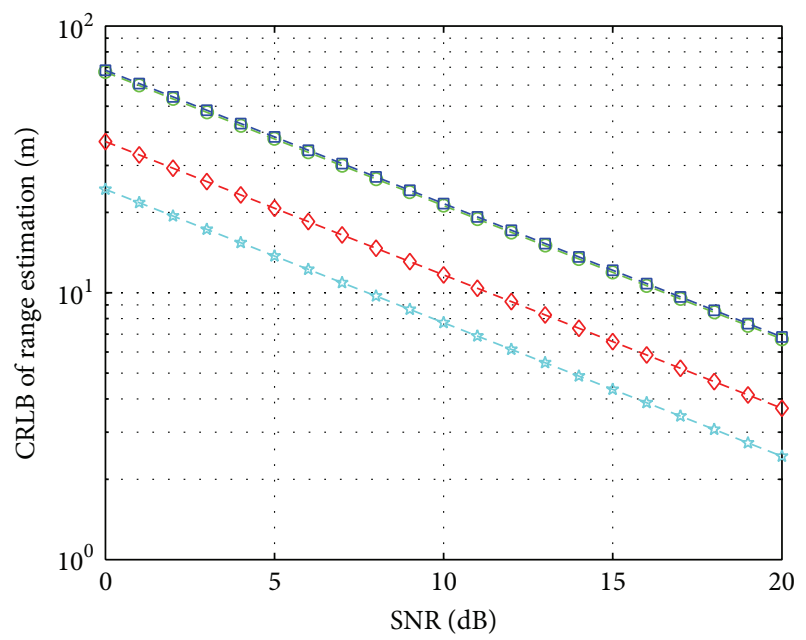

一完 $-\Delta f_{1}=-30 \mathrm{kHz}, \Delta f_{2}=30 \mathrm{kHz}-\ominus-\Delta f_{1}=0 \mathrm{kHz}, \Delta f_{2}=30 \mathrm{kHz}$

$-\diamond-\Delta f_{1}=-15 \mathrm{kHz}, \Delta f_{2}=30 \mathrm{kHz}-$-日 $-\Delta f_{1}=15 \mathrm{kHz}, \Delta f_{2}=40 \mathrm{kHz}$

(b) CRLB for estimating range versus SNR

Figure 5: CRLBs of TS-FDA radar with $M=32$.

this problem, this paper proposes a transmit subaperturing strategy for the FDA radar. In doing so, the range and angle of targets are estimated from the transmit-receive beamforming output. Moreover, we also specialize the CRLB results to the case of temporally white noise and a chirp pulse signal. Extensive simulation results verify the correctness of the derived CRLBs. It is shown that the CRLBs decrease with the increase of the number of elements and frequency increment. The CRLBs can be further improved through three aspects: increasing the number of elements, enhancing the system bandwidth by employing a larger frequency increment, and using transmit subaperturing strategy with more subarrays. 


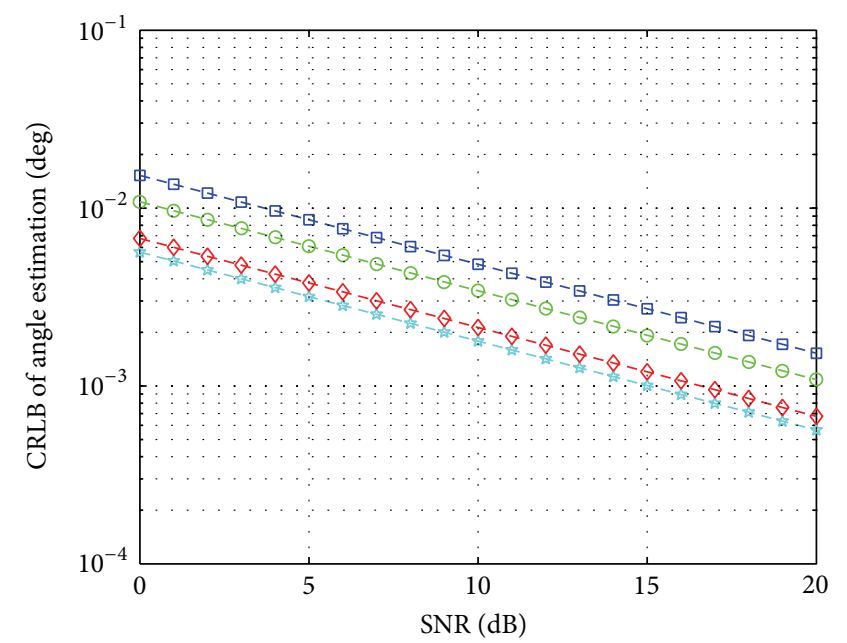

一直一 $\Delta f_{1}=-30 \mathrm{kHz}, \Delta f_{2}=30 \mathrm{kHz} \quad-\ominus-\Delta f_{1}=0 \mathrm{kHz}, \Delta f_{2}=30 \mathrm{kHz}$ $-\diamond-\Delta f_{1}=-15 \mathrm{kHz}, \Delta f_{2}=30 \mathrm{kHz} \quad-\boxminus-\Delta f_{1}=15 \mathrm{kHz}, \Delta f_{2}=40 \mathrm{kHz}$

(a) CRLB for estimating angle versus SNR

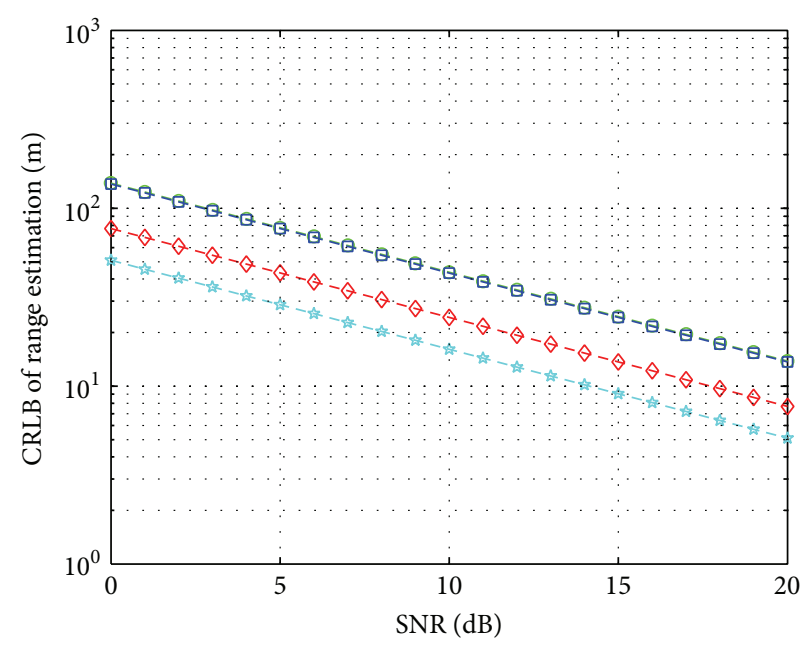

$-\Delta f_{1}=-30 \mathrm{kHz}, \Delta f_{2}=30 \mathrm{kHz} \quad-\ominus-\Delta f_{1}=0 \mathrm{kHz}, \Delta f_{2}=30 \mathrm{kHz}$ $-\diamond-\Delta f_{1}=-15 \mathrm{kHz}, \Delta f_{2}=30 \mathrm{kHz} \quad-\boxminus-\Delta f_{1}=15 \mathrm{kHz}, \Delta f_{2}=40 \mathrm{kHz}$

(b) CRLB for estimating range versus SNR

FIgURE 6: CRLBs of TS-FDA radar with $M=20$.

\section{Appendices}

\section{A. Derive the CRLB for Angle When Range Is Known}

To derive the CRLB, we start with a well-known expression for the FIM under the data model in Section 3. We define the spatial noise covariance matrix as $\mathbf{R}_{\mathbf{n}}=\sigma_{n}^{2} \mathbf{I}_{M}$ and signal-tonoise ratio (SNR) as SNR $=\left|\beta_{0}\right|^{2} / \sigma_{n}^{2}$. Suppose the target range is known, the FIM of $\theta$ is

$$
\begin{aligned}
I_{\theta \theta} & =2 \operatorname{Re}\left\{D_{\theta}^{\mathrm{H}}(\psi)\left(\mathbf{R}_{\mathbf{n}}^{-1}\right) D_{\theta}(\psi)\right\} \\
& =2\left|\beta_{0}\right|^{2} \operatorname{Re}\left\{\frac{\partial \vec{\mu}^{\mathrm{H}}(\psi)}{\partial \theta} \mathbf{R}_{\mathbf{n}}^{-1} \frac{\partial \vec{\mu}(\psi)}{\partial \theta}\right\} .
\end{aligned}
$$
have

For a phased-array radar, there is $\vec{\mu}(\psi)=\beta_{0} \mathbf{a}(\theta)$. We then

$$
\frac{\partial \mathbf{a}(\theta)}{\partial \theta}=-j \frac{2 \pi d \cos (\theta)}{\lambda} \mathbf{D a}(\theta)
$$

where, $\mathbf{D}=\operatorname{diag}[0,1, \ldots, M-1]$ and

$$
\frac{\partial \mathbf{a}^{\mathrm{H}}(\theta)}{\partial \theta} \frac{\partial \mathbf{a}(\theta)}{\partial \theta}=\frac{4 \pi^{2} d^{2} \cos ^{2}(\theta)}{\lambda^{2}} \sum_{m=1}^{M}(m-1)^{2} .
$$

The FIM of the phased-array radar is

$$
I_{\theta \theta_{\text {phased-array }}}=2 \operatorname{SNR} \frac{4 \pi^{2} d^{2} \cos ^{2}(\theta)}{\lambda^{2}} \sum_{m=1}^{M}(m-1)^{2} .
$$

Similarly, for the FDA radar, there is $\vec{\mu}(\psi)=\beta_{0} \mathbf{a}(\theta, r)$. The derivation of $\mathbf{a}(\theta, r)$ with respect to $\theta$ is

$$
\begin{aligned}
& \frac{\partial \mathbf{a}(\theta, r)}{\partial \theta}=-j \frac{2 \pi d \cos (\theta)}{\lambda} \mathbf{D a}(\theta, r) \\
& -j \frac{2 \pi d \Delta f \cos (\theta)}{c} \\
& \times \operatorname{diag}\left[0,1, \ldots,(M-1)^{2}\right] \mathbf{a}(\theta, r) \text {, } \\
& \frac{\partial \mathbf{a}^{\mathrm{H}}(\theta, r)}{\partial \theta} \frac{\partial \mathbf{a}(\theta, r)}{\partial \theta}=4 \pi^{2} d^{2} \cos ^{2}(\theta) \\
& \left\{\frac{\sum_{m=1}^{M}(m-1)^{2}}{\lambda^{2}}\right. \\
& +\frac{(\Delta f)^{2} \sum_{m=1}^{M}(m-1)^{4}}{c^{2}} \\
& \left.+\frac{2 \Delta f \sum_{m=1}^{M}(m-1)^{3}}{\lambda c}\right\} \text {. }
\end{aligned}
$$

Accordingly, the FIM of $\theta$ for the FDA can be expressed as

$$
\begin{aligned}
I_{\theta \theta_{\mathrm{FDA}}}= & 2 \mathrm{SNR} \cdot 4 \pi^{2} d^{2} \cos ^{2}(\theta) \\
& \cdot\left\{\frac{\sum_{m=1}^{M}(m-1)^{2}}{\lambda^{2}}+\frac{(\Delta f)^{2} \sum_{m=1}^{M}(m-1)^{4}}{c^{2}}\right. \\
& \left.+\frac{2 \Delta f \sum_{m=1}^{M}(m-1)^{3}}{\lambda c}\right\} .
\end{aligned}
$$




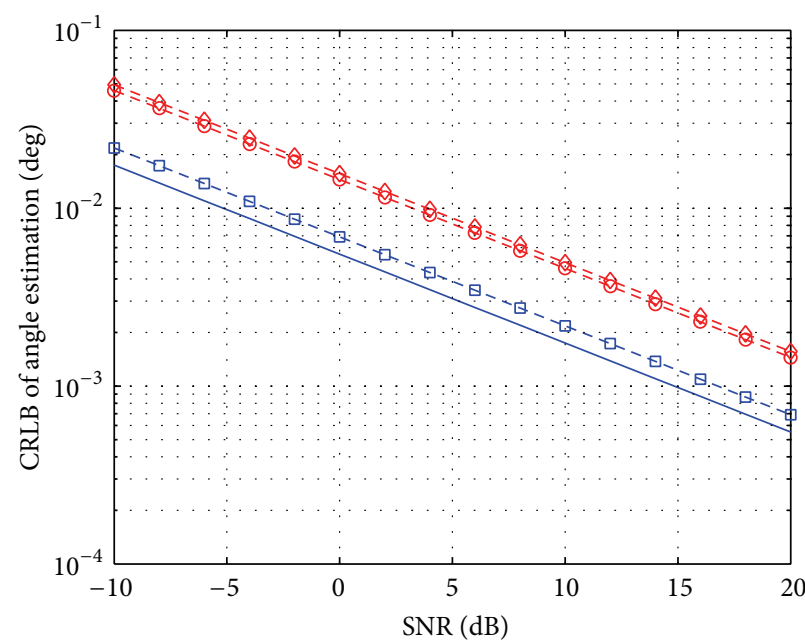

$\begin{array}{ll}-\ominus-M=16, \Delta f=15 \mathrm{kHz} & -M=32, \Delta f=15 \mathrm{kHz} \\ -\vartheta-M=16, \Delta f=30 \mathrm{kHz} & \text {-日-M }=32, \Delta f=30 \mathrm{kHz}\end{array}$

(a) CRLB for estimating angle versus SNR

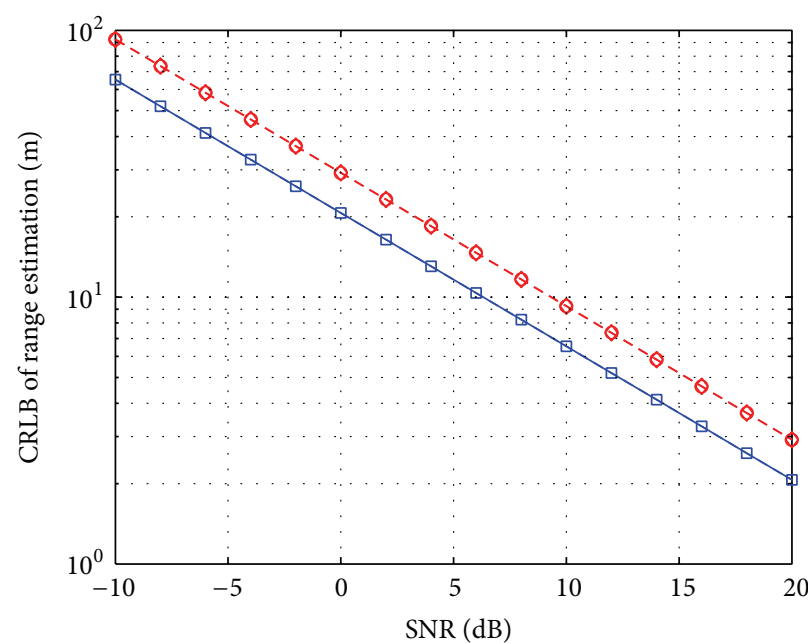

- $\ominus-M=16, \Delta f=15 \mathrm{kHz} \quad-M=32, \Delta f=15 \mathrm{kHz}$

$-\vartheta-M=16, \Delta f=30 \mathrm{kHz} \quad-$ в $-M=32, \Delta f=30 \mathrm{kHz}$

(b) CRLB for estimating range versus SNR

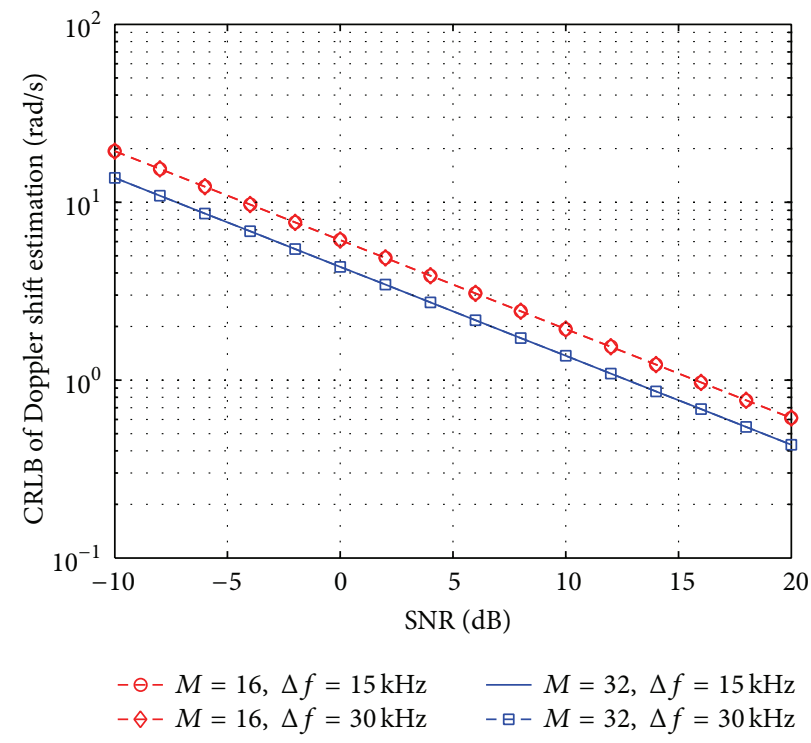

(c) CRLB for Doppler shift versus SNR

Figure 7: General CRLB results of FDA radar

\section{B. Derive the CRLB for Range When Angle Is Known}

Under the data model in Section 3, when the direction $\theta$ is known, the parameter to be estimated is $r$. The FIM of $r$ is

$$
\begin{aligned}
I_{r r_{\mathrm{FDA}}} & =2 \operatorname{Re}\left\{D_{r}^{\mathrm{H}}(\psi)\left(\mathbf{R}_{\mathbf{n}}^{-1}\right) D_{r}(\psi)\right\} \\
& =2\left|\beta_{0}\right|^{2} \operatorname{Re}\left\{\frac{\partial \mathbf{a}^{\mathrm{H}}(\theta, r)}{\partial r} \mathbf{R}_{\mathbf{n}}^{-1} \frac{\partial \mathbf{a}(\theta, r)}{\partial r}\right\} .
\end{aligned}
$$

The derivation of $\mathbf{a}(\theta, r)$ with respect to $r$ for FDA is

$$
\begin{gathered}
\frac{\partial \mathbf{a}(\theta, r)}{\partial r}=j \frac{2 \pi \Delta f}{c} \mathbf{D a}(\theta, r), \\
\frac{\partial \mathbf{a}^{\mathrm{H}}(\theta, r)}{\partial r} \frac{\partial \mathbf{a}(\theta, r)}{\partial r}=\frac{4 \pi^{2} \Delta f^{2}}{c^{2}} \sum_{m=1}^{M}(m-1)^{2} .
\end{gathered}
$$

The FIM of $r$ is thus given by

$$
I_{r r_{\mathrm{FDA}}}=2 \operatorname{SNR} \frac{4 \pi^{2}(\Delta f)^{2}}{c^{2}} \sum_{m=1}^{M}(m-1)^{2} .
$$




\section{Derive the CRLB for Range and Angle}

Under the data model in Section 3, both the angle and range are unknown. The range and angle of targets are estimated jointly. The FIM for parameters $\theta$ and $r$ can be expressed as

$$
\mathbf{I}_{\mathrm{FDA}}=2 \operatorname{Re}\left\{D_{\psi_{i}}^{\mathrm{H}}(\psi)\left(\mathbf{R}_{\mathbf{n}}^{-1}\right) D_{\psi_{j}}(\psi)\right\}=\left[\begin{array}{cc}
I_{\theta \theta} & I_{\theta r} \\
I_{r \theta} & I_{r r}
\end{array}\right] .
$$

We then have

$$
\begin{aligned}
\mathbf{I}_{\mathrm{FDA}}= & 2\left|\beta_{0}\right|^{2} \\
& \times\left[\begin{array}{ll}
\frac{\partial \vec{\mu}^{\mathrm{H}}(\psi)}{\partial \theta} \mathbf{R}_{\mathbf{n}}^{-1} \frac{\partial \vec{\mu}(\psi)}{\partial \theta} & \frac{\partial \vec{\mu}^{\mathrm{H}}(\psi)}{\partial r} \mathbf{R}_{\mathbf{n}}^{-1} \frac{\partial \vec{\mu}(\psi)}{\partial \theta} \\
\frac{\partial \vec{\mu}^{\mathrm{H}}(\psi)}{\partial \theta} \mathbf{R}_{\mathbf{n}}^{-1} \frac{\partial \vec{\mu}(\psi)}{\partial r} & \frac{\partial \vec{\mu}^{\mathrm{H}}(\psi)}{\partial r} \mathbf{R}_{\mathbf{n}}^{-1} \frac{\partial \vec{\mu}(\psi)}{\partial r}
\end{array}\right] .
\end{aligned}
$$

Since $\left(\partial \vec{\mu}^{\mathrm{H}}(\psi) / \partial r\right) \mathbf{R}_{\mathbf{n}}^{-1}(\partial \vec{\mu}(\psi) / \partial \theta)=\left(\partial \vec{\mu}^{\mathrm{H}}(\psi) /\right.$ $\partial \theta) \mathbf{R}_{\mathbf{n}}^{-1}(\partial \vec{\mu}(\psi) / \partial r)$, then $I_{\theta r}=I_{r \theta}$. We can get

$$
\begin{aligned}
I_{\theta \theta}= & 2\left|\beta_{0}\right|^{2} \frac{\partial \vec{\mu}^{\mathrm{H}}(\psi)}{\partial \theta} \mathbf{R}_{\mathbf{n}}^{-1} \frac{\partial \vec{\mu}(\psi)}{\partial \theta} \\
= & 8 \pi^{2} d^{2} \cos ^{2}(\theta) \\
& \times \operatorname{SNR}\left\{\frac{\sum_{m=1}^{M}(m-1)^{2}}{\lambda^{2}}+\frac{(\Delta f)^{2} \sum_{m=1}^{M}(m-1)^{4}}{c^{2}}\right. \\
& \left.+\frac{2 \Delta f \sum_{m=1}^{M}(m-1)^{3}}{\lambda c}\right\}, \\
I_{\theta r}= & 2\left|\beta_{0}\right|^{2} \frac{\partial \vec{\mu}^{\mathrm{H}}(\psi)}{\partial \theta} \mathbf{R}_{\mathbf{n}}^{-1} \frac{\partial \vec{\mu}(\psi)}{\partial r} \\
= & -8 \pi^{2} d \Delta f \cos (\theta) \\
& \times \mathrm{SNR}\left[\frac{\sum_{m=1}^{M}(m-1)^{2}}{\lambda c}+\frac{\Delta f \sum_{m=1}^{M}(m-1)^{3}}{c^{2}}\right], \\
I_{r r}= & 2\left|\beta_{0}\right|^{2} \frac{\partial \vec{\mu}^{\mathrm{H}}(\psi)}{\partial r} \mathbf{R}_{\mathbf{n}}^{-1} \frac{\partial \vec{\mu}(\psi)}{\partial r} \\
= & \mathrm{SNR} \frac{8 \pi^{2}(\Delta f)^{2}}{c^{2}} \sum_{m=1}^{M}(m-1)^{2} .
\end{aligned}
$$

Since $\mathrm{CRLB}_{\theta \theta_{\mathrm{FDA}}}=\left[\mathbf{I}_{\mathrm{FDA}}^{-1}\right]_{1,1}, \mathrm{CRLB}_{r r_{\mathrm{FDA}}}=\left[\mathbf{I}_{\mathrm{FDA}}^{-1}\right]_{2,2}$. The expressions for the CRLB of angle and range estimations given in (14) can be obtained by substituting (C.3) into (C.2).

\section{General CRLB Results}

Under the data model in Section 4, we derive the continuous CRLB expressions for temporally and spatially white noise and denote SNR $=|\beta|^{2}\|\mathbf{s}\| / \sigma_{n}^{2}$ with $\|\mathbf{s}\|$ being the power. We also start with FIM:

$$
\mathbf{I}_{\kappa_{i} \kappa_{j}}=2 \operatorname{Re}\left\{D_{\kappa_{i}}^{\mathrm{H}}(\kappa)\left(\mathbf{C}_{\mathbf{n}}^{-1} \otimes \mathbf{R}_{\mathbf{n}}^{-1}\right) D_{\kappa_{j}}(\kappa)\right\},
$$

where $\kappa_{i}$ is the $i$ th element of $\kappa$ and $D_{\kappa_{i}}(\psi)=\partial \vec{\mu}(\kappa) / \partial \kappa_{i}$. Consider

$$
\mathbf{I}_{\mathrm{FDA}}=\left[\begin{array}{ccc}
I_{\beta \beta} & I_{\theta \beta}^{T} & I_{\eta \beta}^{T} \\
I_{\theta \beta} & I_{\theta \theta} & I_{\eta \theta}^{T} \\
I_{\eta \beta} & I_{\eta \theta} & I_{\eta \eta}
\end{array}\right] .
$$

For clarity, we rewrite Fisher's information matrix I as

$$
\mathbf{I}_{\mathrm{FDA}}=\left[\begin{array}{cc}
\mathbf{A} & \mathbf{U} \\
\mathbf{V} & \mathbf{B}
\end{array}\right]
$$

where

$$
\begin{aligned}
& \mathbf{V}=\mathbf{U}^{T}, \\
& \mathbf{V}=\left[\begin{array}{l}
I_{\theta \beta} \\
I_{\eta \beta}
\end{array}\right], \\
& \mathbf{B}=\left[\begin{array}{ll}
I_{\theta \theta} & I_{\eta \theta}^{T} \\
I_{\eta \theta} & I_{\eta \eta}
\end{array}\right] .
\end{aligned}
$$

According to the matrix inversion lemma, the inverse matrix of $\mathbf{I}_{\mathrm{FDA}}$ is

$$
\mathbf{I}_{\mathrm{FDA}}^{-1}=\left[\begin{array}{cc}
\left(\mathbf{A}-\mathbf{U B}^{-1} \mathbf{V}\right)^{-1} & -\mathbf{A}^{-1} \mathbf{U}\left(\mathbf{B}-\mathbf{V A}^{-1} \mathbf{U}\right)^{-1} \\
-\mathbf{B}^{-1} \mathbf{V}\left(\mathbf{A}-\mathbf{U B}^{-1} \mathbf{V}\right)^{-1} & \left(\mathbf{B}-\mathbf{V A}^{-1} \mathbf{U}\right)^{-1}
\end{array}\right],
$$

where the submatrix $\left(\mathbf{A}-\mathbf{U B}^{-1} \mathbf{V}\right)^{-1}$ corresponds to the CRLB of $\beta$ which is nuisance and $\left(\mathbf{B}-\mathbf{V A}^{-1} \mathbf{U}\right)^{-1}$ corresponds to the CRLB of $\theta$ and $\eta=\left[r, \Omega_{D}\right]^{T}$ which are of interest.

$$
\begin{aligned}
\mathrm{CRLB}_{\theta \eta, \theta \eta_{\mathrm{FDA}}} & =\left(\mathbf{B}-\mathbf{V A}^{-1} \mathbf{U}\right)^{-1} \\
& =\left\{\left[\begin{array}{cc}
I_{\theta \theta} & I_{\eta \theta}^{T} \\
I_{\eta \theta} & I_{\eta \eta}
\end{array}\right]-\left[\begin{array}{c}
I_{\theta \beta} \\
I_{\eta \beta}
\end{array}\right] I_{\beta \beta}^{-1}\left[\begin{array}{ll}
I_{\theta \beta}^{T} & I_{\eta \beta}^{T}
\end{array}\right]\right\}^{-1},
\end{aligned}
$$

where

$$
\begin{aligned}
& I_{\beta \beta}=2 \cdot\left[\begin{array}{cc}
\frac{\|\mathbf{s}\| \cdot M}{\sigma_{n}^{2}} & 0 \\
0 & \frac{\|\mathbf{s}\| \cdot M}{\sigma_{n}^{2}}
\end{array}\right], \\
& I_{\theta \beta}=2 \cdot \operatorname{Re}\left\{\left[\begin{array}{ll}
1 & j
\end{array}\right] \otimes \frac{\|\mathbf{s}\|}{\sigma_{n}^{2}} \cdot \beta^{*} A_{1}\right\}, \\
& I_{\eta \beta}=2 \cdot \operatorname{Re}\left\{\left[\begin{array}{ll}
1 & j
\end{array}\right] \otimes \frac{\beta^{*}}{\sigma_{w}^{2}} \cdot\left[M \cdot A_{3}+\|\mathbf{s}\| \cdot A_{4}\right]\right\}, \\
& I_{\eta \eta}=2 \cdot \operatorname{Re}\left\{\frac{|\beta|^{2}}{\sigma_{n}^{2}}\left[M \cdot A_{6}+A_{3}^{\mathrm{H}} A_{4}+A_{3} A_{4}^{\mathrm{H}}+\|\mathbf{s}\| \cdot A_{7}\right]\right\},
\end{aligned}
$$



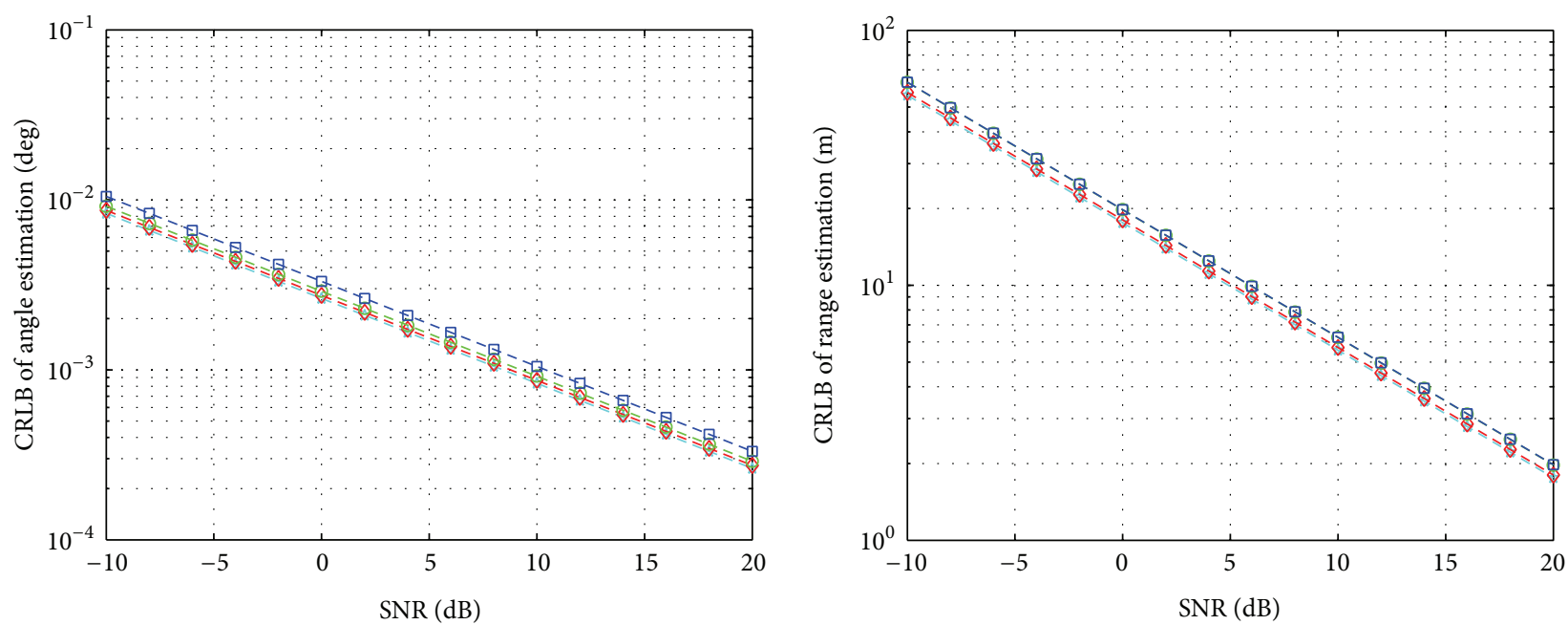

一角 $-\Delta f_{1}=-30 \mathrm{kHz}, \Delta f_{2}=30 \mathrm{kHz} \quad-\ominus-\Delta f_{1}=0 \mathrm{kHz}, \Delta f_{2}=30 \mathrm{kHz}$ $-\diamond-\Delta f_{1}=-15 \mathrm{kHz}, \Delta f_{2}=30 \mathrm{kHz} \quad-\boxminus-\Delta f_{1}=15 \mathrm{kHz}, \Delta f_{2}=40 \mathrm{kHz}$

(a) CRLB for estimating angle versus SNR

一会 $-\Delta f_{1}=-30 \mathrm{kHz}, \Delta f_{2}=30 \mathrm{kHz} \quad-\ominus-\Delta f_{1}=0 \mathrm{kHz}, \Delta f_{2}=30 \mathrm{kHz}$ $-\ominus-\Delta f_{1}=-15 \mathrm{kHz}, \Delta f_{2}=30 \mathrm{kHz} \quad-$ - $-\Delta f_{1}=15 \mathrm{kHz}, \Delta f_{2}=40 \mathrm{kHz}$

(b) CRLB for estimating range versus SNR

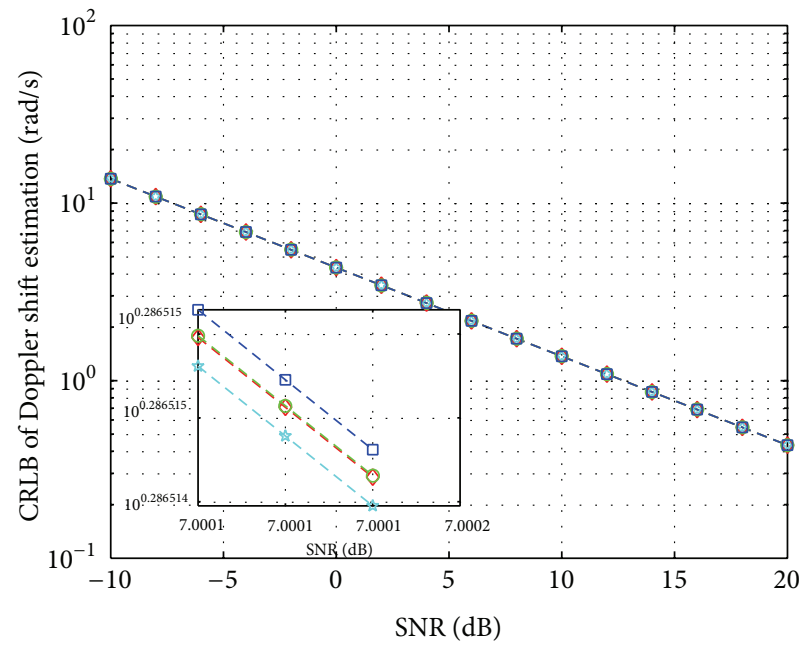

一 $-\Delta f_{1}=-30 \mathrm{kHz}, \Delta f_{2}=30 \mathrm{kHz} \quad-\ominus-\Delta f_{1}=0 \mathrm{kHz}, \Delta f_{2}=30 \mathrm{kHz}$
$-\diamond-\Delta f_{1}=-15 \mathrm{kHz}, \Delta f_{2}=30 \mathrm{kHz} \quad-$ - $-\Delta f_{1}=15 \mathrm{kHz}, \Delta f_{2}=40 \mathrm{kHz}$

(c) CRLB for Doppler shift versus SNR

FIGURE 8: General CRLB results of TS-FDA radar with $M=32$.

$$
\begin{aligned}
& \left.I_{\theta \theta}-\left(I_{\theta \beta} I_{\theta \beta}^{T}\right) \frac{\sigma_{n}^{2}}{\|\mathbf{s}\| M}=2 \frac{\|\mathbf{s}\||\beta|^{2}}{\sigma_{n}^{2}}\left(A_{2}-\frac{A_{1} A_{1}^{\mathrm{H}}}{M}\right), \quad \text { (D. } 80\right) \\
& I_{\eta \theta}-\left(I_{\eta \beta} I_{\theta \beta}^{T}\right) \frac{\sigma_{n}^{2}}{\|\mathbf{s}\| M}=2 \frac{\|\mathbf{s}\||\beta|^{2}}{\sigma_{n}^{2}}\left(A_{5}-\frac{A_{4} A_{1}^{\mathrm{H}}}{M}\right), \quad \text { (D. } \\
& I_{\eta \eta}-\left(I_{\eta \beta} I_{\eta \beta}^{T}\right) \frac{\sigma_{n}^{2}}{\|\mathbf{s}\| M} \\
& \quad=2 \frac{|\beta|^{2}\|\mathbf{s}\|}{\sigma_{n}^{2}}\left(\frac{M A_{6}}{\|\mathbf{s}\|}+A_{7}-\frac{M A_{3} A_{3}^{\mathrm{H}}}{\|\mathbf{s}\|^{2}}-\frac{A_{4} A_{4}^{\mathrm{H}}}{M}\right),
\end{aligned}
$$

$$
\begin{aligned}
A_{1}=-j & \frac{2 \pi d \cos (\theta) \sum_{m=1}^{M}(m-1)}{\lambda} \\
& \left.+\frac{2 \pi d \Delta f \cos (\theta) \sum_{m=1}^{M}(m-1)^{2}}{c}\right),
\end{aligned}
$$

$$
\begin{aligned}
A_{2}= & 4 \pi^{2} d^{2} \cos ^{2}(\theta) \\
& \cdot\left\{\frac{\sum_{m=1}^{M}(m-1)^{2}}{\lambda^{2}}+\frac{(\Delta f)^{2} \sum_{m=1}^{M}(m-1)^{4}}{c^{2}}\right. \\
& \left.+\frac{2 \Delta f \sum_{m=1}^{M}(m-1)^{3}}{\lambda c}\right\},
\end{aligned}
$$



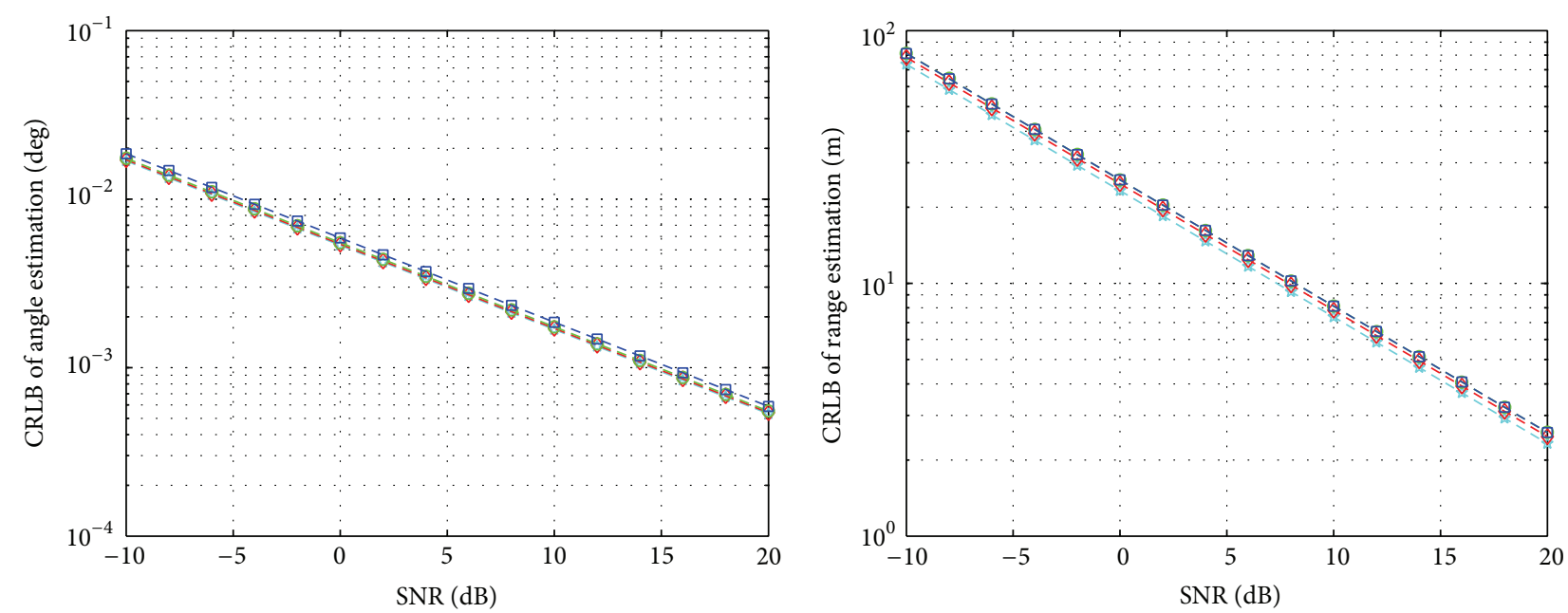

一古 $-\Delta f_{1}=-30 \mathrm{kHz}, \Delta f_{2}=30 \mathrm{kHz} \quad-\ominus-\Delta f_{1}=0 \mathrm{kHz}, \Delta f_{2}=30 \mathrm{kHz} \quad$ - 分 $-\Delta f_{1}=-30 \mathrm{kHz}, \Delta f_{2}=30 \mathrm{kHz} \quad-\ominus-\Delta f_{1}=0 \mathrm{kHz}, \Delta f_{2}=30 \mathrm{kHz}$ $-\ominus-\Delta f_{1}=-15 \mathrm{kHz}, \Delta f_{2}=30 \mathrm{kHz} \quad-$ - $-\Delta f_{1}=15 \mathrm{kHz}, \Delta f_{2}=40 \mathrm{kHz} \quad-\ominus-\Delta f_{1}=-15 \mathrm{kHz}, \Delta f_{2}=30 \mathrm{kHz} \quad-\theta-\Delta f_{1}=15 \mathrm{kHz}, \Delta f_{2}=40 \mathrm{kHz}$

(a) CRLB for estimating angle versus SNR

(b) CRLB for estimating range versus SNR

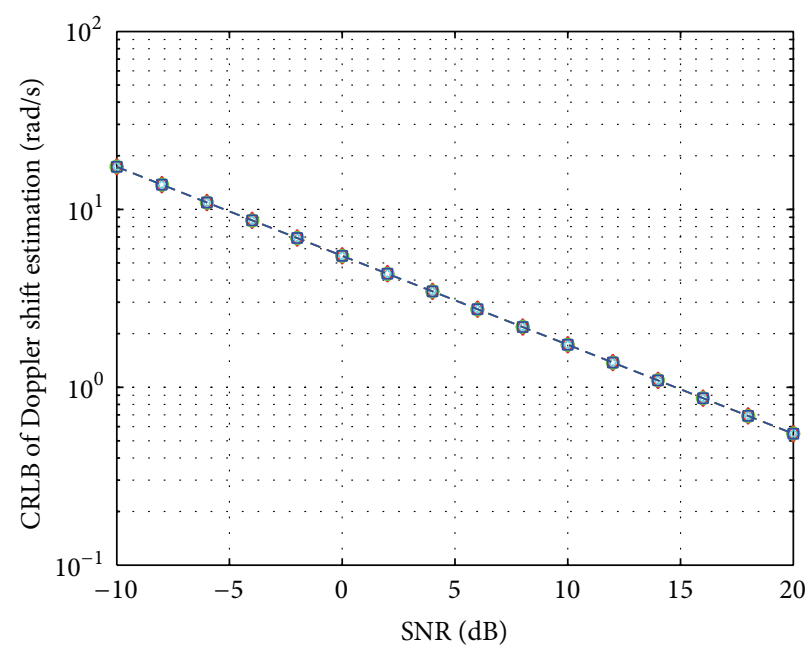

一 - $\Delta f_{1}=-30 \mathrm{kHz}, \Delta f_{2}=30 \mathrm{kHz}-\ominus-\Delta f_{1}=0 \mathrm{kHz}, \Delta f_{2}=30 \mathrm{kHz}$

$-\diamond-\Delta f_{1}=-15 \mathrm{kHz}, \Delta f_{2}=30 \mathrm{kHz} \quad-\boxminus-\Delta f_{1}=15 \mathrm{kHz}, \Delta f_{2}=40 \mathrm{kHz}$

(c) CRLB for Doppler shift versus SNR

FIGURE 9: General CRLB results of TS-FDA radar with $M=20$.

$$
\begin{gathered}
A_{3}=\left[\int_{-\infty}^{\infty} s(t-\tau) \frac{d s(t-\tau)^{\mathrm{H}}}{d t} d t \int_{-\infty}^{\infty} t|s(t-\tau)|^{2} d t\right]^{T}, \\
A_{4}=\left[\frac{2 \pi \Delta f \sum_{m=1}^{M}(m-1)}{c} 0\right]^{T},
\end{gathered}
$$

$A_{5}=\left[-4 \pi^{2} d \Delta f \cos (\theta)\left[\frac{\sum_{m=1}^{M}(m-1)^{2}}{12 \lambda c}+\frac{\Delta f \sum_{m=1}^{M}(m-1)^{3}}{12 c^{2}}\right] 0\right]^{T}$,

$$
A_{6}=\left[\begin{array}{cc}
\int_{-\infty}^{\infty}\left|\frac{d s(t-\tau)}{d t}\right|^{2} d t & \int_{-\infty}^{\infty} t s^{\mathrm{H}}(t-\tau) \frac{d s(t-\tau)}{d t} d t \\
\int_{-\infty}^{\infty} t s(t-\tau) \frac{d s(t-\tau)^{\mathrm{H}}}{d t} d t & \int_{-\infty}^{\infty} t^{2}|s(t-\tau)|^{2} d t
\end{array}\right],
$$

$$
A_{7}=\left[\begin{array}{cc}
\frac{4 \pi^{2}(\Delta f)^{2}}{c^{2}} \sum_{m=1}^{M}(m-1)^{2} & 0 \\
0 & 0
\end{array}\right]
$$

\section{Conflict of Interests}

The authors declare that there is no conflict of interests regarding the publication of this paper.

\section{Acknowledgments}

The work described in this paper was supported in part by the National Natural Science Foundation of China under Grant 
41101317, the Program for New Century Excellent Talents in University under Grant NCET-12-0095, Sichuan Province Science Fund for Distinguished Young Scholars under Grant 2013JQ0003, and Fundamental Research Fund for the Central Universities.

\section{References}

[1] F. Bandiera, M. Mancino, and G. Ricci, "Localization strategies for multiple point-like radar targets," IEEE Transactions on Signal Processing, vol. 60, no. 12, pp. 6708-6712, 2012.

[2] D. R. Fuhrmann, J. P. Browning, and M. Rangaswamy, "Signaling strategies for the hybrid MIMO phased-array radar," IEEE Journal on Selected Topics in Signal Processing, vol. 4, no. 1, pp. 66-78, 2010.

[3] S. Sen and A. Nehorai, "Adaptive OFDM radar for target detection in multipath scenarios," IEEE Transactions on Signal Processing, vol. 59, no. 1, pp. 78-90, 2011.

[4] M. Compagnoni, P. Bestagini, F. Antonacci, A. Sarti, and S. Tubaro, "Localization of acoustic sources through the fitting of propagation cones using multiple independent arrays," IEEE Transactions on Audio, Speech, and Language Processing, vol. 20, no. 7, pp. 1964-1975, 2012.

[5] P. Antonik, M. C. Wicks, H. D. Griffiths, and C. J. Baker, "Frequency diverse array radars," in Proceedings of the IEEE Radar Conference (RADAR '06), pp. 215-217, Verona, NY, USA, April 2006.

[6] P. Antonik, M. C. Wicks, H. D. Griffiths, and C. J. Baker, "Multimission multi-mode waveform diversity," in Proceedings of the IEEE Radar Conference (RADAR '06), pp. 580-582, Verona, NY, USA, April 2006.

[7] P. Antonik, H. D. Griffiths, and C. J. Baker, "Range dependent beamforming using element level waveform diversity," in Proceedings of the International Waveform Diversity and Design Conference, pp. 1-4, Las Vegas, Nev, USA, January 2006.

[8] P. Baizert, T. B. Hale, M. A. Temple, and M. C. Wicks, "Forwardlooking radar GMTI benefits using a linear frequency diverse array," Electronics Letters, vol. 42, no. 22, pp. 1311-1312, 2006.

[9] B. W. Jung, R. S. Adve, and J. Chun, "Frequency diversity in multistatic radars," in Proceedings of the IEEE Radar Conference (RADAR '08), pp. 1-6, Rome, Italy, May 2008.

[10] M. Secmen, S. Demir, A. Hizal, and T. Eker, "Frequency diverse array antenna with periodic time modulated pattern in range and angle," in Proceedings of the IEEE Radar Conference (RADAR '07), pp. 427-430, Boston, Mass, USA, April 2007.

[11] J. Huang, K. F. Tong, and C. J. Baker, "Frequency diverse array with beam scanning feature," in Proceedings of the IEEE Antennas and Propagation Conference (AP-S '08), pp. 1-4, San Diego, Calif, USA, July 2008.

[12] T. Higgins and S. D. Blunt, "Analysis of range-angle coupled beamforming with frequency-diverse chirps," in Proceedings of the International Waveform Diversity and Design Conference (WDD '09), pp. 140-144, Orlando, Fla, USA, February 2009.

[13] J. Farooq, M. A. Temple, and M. A. Saville, "Application of frequency diverse arrays to synthetic aperture radar imaging," in Proceedings of the International Conference on Electromagnetics in Advanced Applications (ICEAA '07), pp. 447-449, Torino, Italy, September 2007.

[14] J. Farooq, M. A. Temple, and M. A. Saville, "Exploiting frequency diverse array processing to improve SAR image resolution," in Proceedings of the IEEE Radar Conference (RADAR '08), pp. 1-5, Rome, Italy, May 2008.
[15] W. Q. Wang, "Phased-MIMO radar with frequency diversity for rangedependent beamforming," IEEE Sensors Journal, vol. 13, no. 8, pp. 1320-1328, 2013.

[16] A. L. Swindlehurst and P. Stoica, "Maximum likelihood methods in radar array signal processing," Proceedings of the IEEE, vol. 86, no. 2, pp. 421-441, 1998.

[17] J. Ward, "Cramér-Rao bounds for target angle and Doppler estimation with space-time adaptive processing radar," in Proceedings of the 29th Asilomar Conference on Signals, Systems and Computers, pp. 1198-1202, Pacific Grove, Calif, USA, November 1995.

[18] A. Dogandzic and A. Nehorai, "Cramér-Rao bounds for estimating range, velocity, and direction with an active array," IEEE Transactions on Signal Processing, vol. 49, no. 6, pp. 1122-1137, 2001.

[19] A. Dogandzic and A. Nehorai, "Estimating range, velocity, and direction with a radar array," in Proceedings of the IEEE International Conference on Acoustics, Speech, and Signal Processing (ICASSP '99), pp. 2773-2776, Phoenix, Ariz, USA, March 1999.

[20] J. J. Zhang, G. Maalouli, A. P. Suppappola, and D. Morrell, "Cramér-Rao lower bounds for the joint estimation of target attributes using MIMO radar," in Proceedings of the International Waveform Diversity and Design Conference (WDD '09), pp. 103-107, Orlando, Fla, USA, February 2009.

[21] A. Hassanien, S. A. Vorobyov, and A. B. Gershman, "Moving target parameters estimation in noncoherent MIMO radar systems," IEEE Transactions on Signal Processing, vol. 60, no. 5, pp. 2354-2361, 2012.

[22] Q. He, R. S. Blum, and A. M. Haimovich, "Noncoherent MIMO radar for location and velocity estimation: more antennas means better performance," IEEE Transactions on Signal Processing, vol. 58, no. 7, pp. 3661-3680, 2010.

[23] S. M. Kay, Fundamentals of Statistical Signal Processing: Estimation Theory, vol. 1, Pearson, London, UK, 2nd edition, 2011.

[24] H. L. van Trees, Optimum Array Processing, John Wiley \& Sons, New York, NY, USA, 2002.

[25] D. Wilcox and M. Sellathurai, "On MIMO radar subarrayed transmit beamforming," IEEE Transactions on Signal Processing, vol. 60, no. 4, pp. 2076-2081, 2012.

[26] W. Q. Wang and H. Z. Shao, "Range-angle localization of targets by a double-pulse frequency diverse array radar," IEEE Journal on Selected Topics in Signal Processing, vol. 8, no. 1, pp. 106-114, 2014.

[27] S. Gogineni and A. Nehorai, "Target estimation using sparse modeling for distributed MIMO radar," IEEE Transactions on Signal Processing, vol. 59, no. 11, pp. 5315-5325, 2011.

[28] T. Li and A. Nehorai, "Maximum likelihood direction finding in spatially colored noise fields using sparse sensor arrays," IEEE Transactions on Signal Processing, vol. 59, no. 3, pp. 1048-1062, 2011.

[29] S. Sen, "OFDM radar space-time adaptive processing by exploiting spatio-temporal sparsity," IEEE Transactions on Signal Processing, vol. 61, no. 1, pp. 118-130, 2013.

[30] J. J. Blanz, A. Papathanassiou, M. Haardt, I. Furió, and P. W. Baier, "Smart antennas for combined DOA and joint channel estimation in time-slotted CDMA mobile radio systems with joint detection," IEEE Transactions on Vehicular Technology, vol. 49, no. 2, pp. 293-306, 2000.

[31] P. N. Pathirana, S. C. K. Herath, and A. V. Savkin, "Multitarget tracking via space transformations using a single frequency continuous wave radar," IEEE Transactions on Signal Processing, vol. 60, no. 10, pp. 5217-5229, 2012. 
[32] P. H. Leong, T. D. Abhayapala, and T. A. Lamahewa, "Multiple target localization using wideband echo chirp signals," IEEE Transactions on Signal Processing, vol. 61, no. 16, pp. 4077-4089, 2013.

[33] L. Zhuang, X. Liu, and W. Yu, "Precisely beam steering for frequency diverse arrays based on frequency offset selection," in Proceedings of the International Radar Conference (RADAR '09), pp. 1-4, Bordeaux, France, December 2009. 

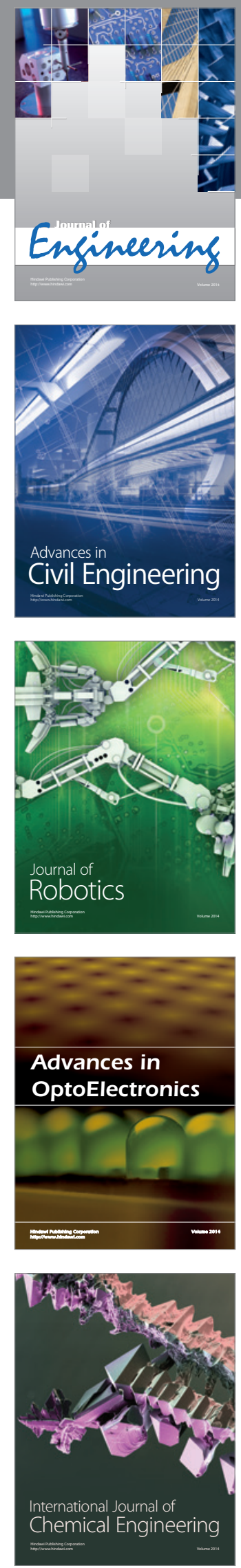

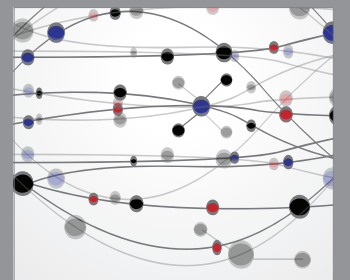

The Scientific World Journal
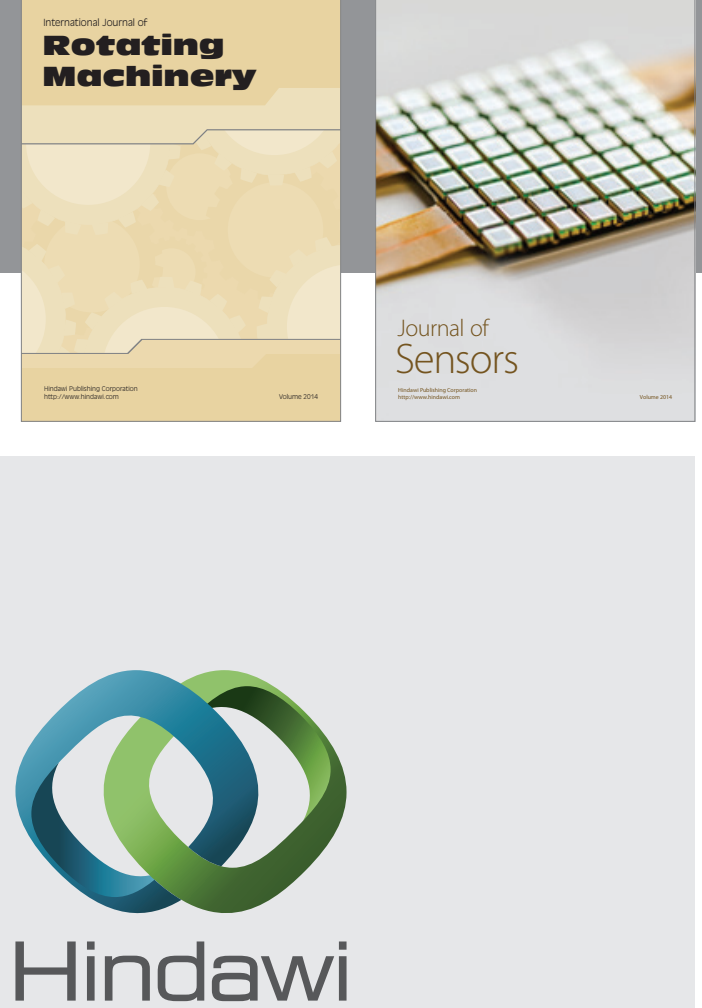

Submit your manuscripts at http://www.hindawi.com
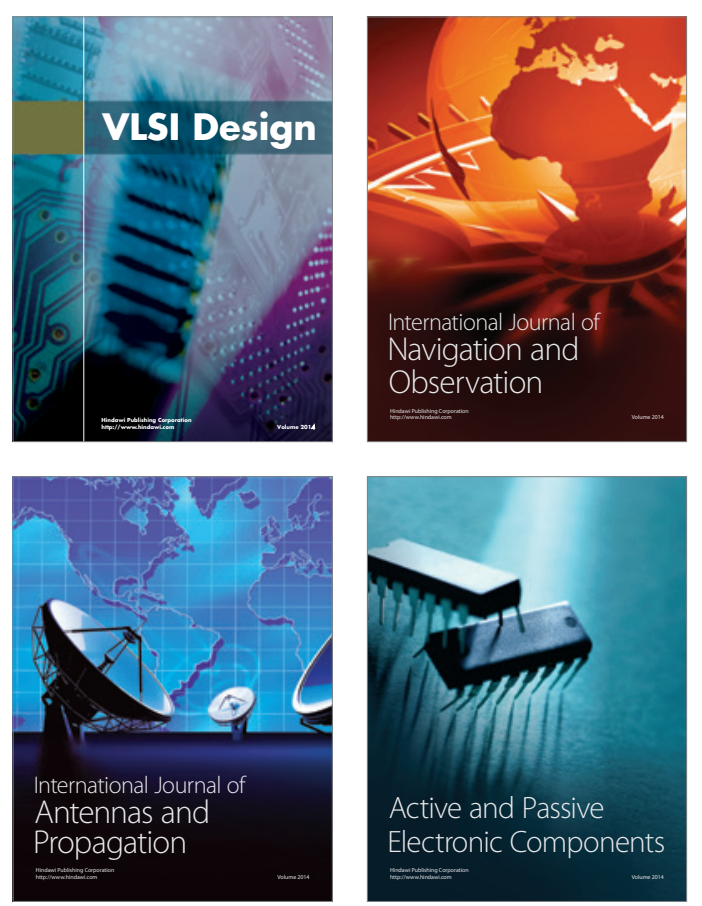
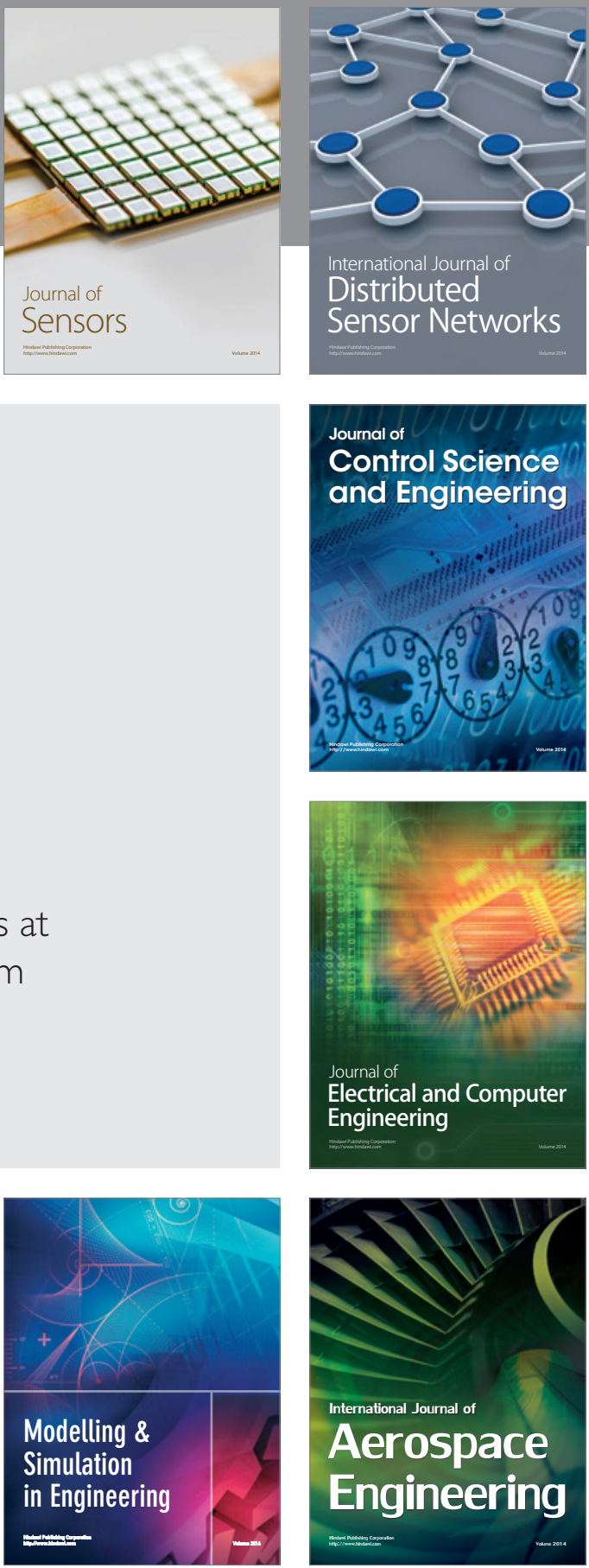

Journal of

Control Science

and Engineering
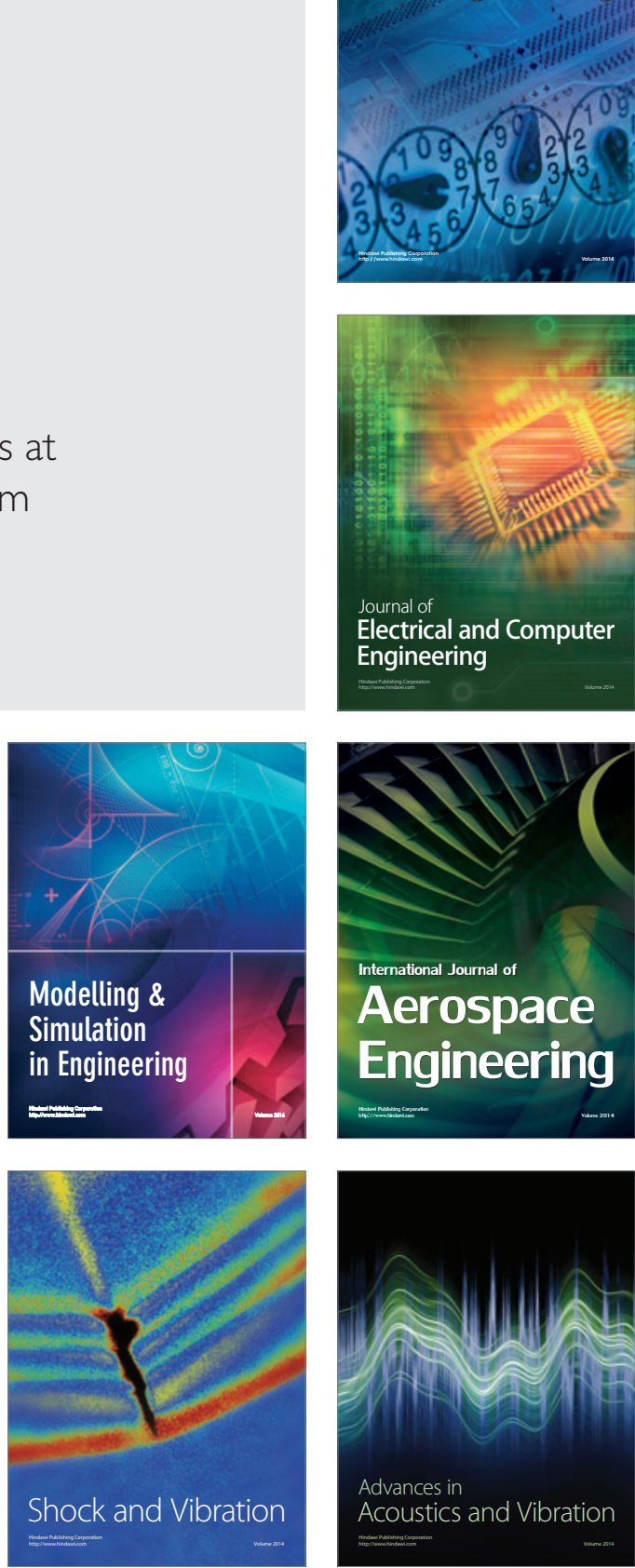